DOI 10.4171/JEMS/191

Artur Avila $\cdot$ Svetlana Jitomirskaya

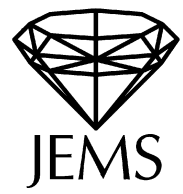

\title{
Almost localization and almost reducibility
}

\author{
Received April 17, 2008
}

\begin{abstract}
We develop a quantitative version of Aubry duality and use it to obtain several sharp estimates for the dynamics of Schrödinger cocycles associated to a non-perturbatively small analytic potential and Diophantine frequency. In particular, we establish the full version of Eliasson's reducibility theory in this regime (our approach actually leads to improvements even in the perturbative regime: we are able to show, for all energies, "almost reducibility" in some band of analyticity). We also prove 1/2-Hölder continuity of the integrated density of states. For the almost Mathieu operator, our results hold through the entire regime of subcritical coupling and imply also the dry version of the Ten Martini Problem for the relevant parameters.
\end{abstract}

Keywords. Quasiperiodic Schrödinger operators, Anderson localization, reducibility, absolutely continuous spectrum

\section{Introduction}

This work is concerned with quasiperiodic Schrödinger operators $H=H_{\lambda v, \alpha, \theta}$ defined on $l^{2}(\mathbb{Z})$ by

$$
(H u)_{n}=u_{n+1}+u_{n-1}+\lambda v(\theta+n \alpha) u_{n}
$$

where $v: \mathbb{R} / \mathbb{Z} \rightarrow \mathbb{R}$ is the potential, $\lambda \in \mathbb{R}$ is the coupling constant, $\alpha \in \mathbb{R} \backslash \mathbb{Q}$ is the frequency and $\theta \in \mathbb{R}$ is the phase. The central (and in some sense most important) example is given by the almost Mathieu operator, when $v(x)=2 \cos 2 \pi x$.

Except where otherwise noted, below we assume the frequency $\alpha$ to be Diophantine in the usual sense, and $v$ analytic.

An important feature of quasiperiodic operators is that the family $\left\{H_{\lambda v, \alpha, \theta}\right\}_{\lambda \in \mathbb{R}}$ undergoes a so called metal-insulator transition when $|\lambda|$ is changed from small to large. Loosely speaking, large $\lambda$ correspond to the insulator phase, with positive Lyapunov exponents and related localization type effects, while small $\lambda$ leads to a metallic phase, with

A. Avila: CNRS UMR 7586, Institut de Mathématiques de Jussieu, 175 rue de Chevaleret, 75013 Paris, France, and IMPA, Estrada Dona Castorina, 22460-320 Rio de Janeiro, Brazil; e-mail: artur@math.sunysb.edu

S. Jitomirskaya: Department of Mathematics, University of California, Irvine, CA 92697-3875, USA; e-mail: szhitomi@uci.edu

Mathematics Subject Classification (2010): Primary 47B36, 37C55; Secondary 39A70, 47A10, 47B39, 81Q10 
zero Lyapunov exponents and good transport properties. Here we are interested in the metallic regime, and therefore in small couplings.

One should distinguish between two possible regimes of small $|\lambda|$ (similar considerations can be applied to the analysis of large coupling). One is perturbative, meaning that the smallness condition on $|\lambda|$ depends not only on the potential $v$, but also on the frequency $\alpha$ : the key resulting limitation is that the analysis at a given coupling, however small, has to exclude a positive Lebesgue measure set of $\alpha$. Such exclusions are inherent to the KAM-type methods that have been traditionally used in this context. The other, stronger regime is called non-perturbative, meaning that the smallness condition on $|\lambda|$ only depends on the potential, leading to results that hold for almost every $\alpha$. Let us stress that, in certain related contexts (multifrequency modifications of (1.1)), perturbative results do fail to extend to the non-perturbative regime (see Remark 1.1).

In [E], Eliasson obtained a very precise description of operators [1.1] in the case of small analytic potentials in the perturbative regime. He proved fine estimates on all solutions of the eigenvalue equation $H u=E u$ for $E$ in the spectrum, concluding that most, but not all, are analytic Bloch waves, i.e. quasiperiodic and analytic in the hull. In his context, the problem of existence of analytic Bloch waves can be restated (and is indeed treated) as a dynamical systems problem, of reducibility of the associated cocycle (see $\$ 1.2$. His method is based on a sophisticated KAM scheme, which avoids the limitations of earlier KAM methods (that go back to the work of Dinaburg-Sinai [DiS] and that excluded parts of the spectrum from consideration).

More recently, a less precise analysis of small analytic potentials has been carried out through the non-perturbative regime in $[\overline{\mathrm{J}}]$ and $[\mathrm{BJ} 1]$. One feature of those results is that most of the analysis is concerned with the dual model $\hat{H}=\hat{H}_{\lambda v, \alpha, \theta}$ defined on $l^{2}(\mathbb{Z})$ by

$$
(\hat{H} \hat{u})_{n}=\sum \lambda \hat{v}_{k} \hat{u}_{n-k}+2 \cos (2 \pi \theta+n \alpha) \hat{u}_{n},
$$

where $\hat{v}_{k}$ are the Fourier coefficients of $v(x)=\sum \hat{v}_{k} e^{2 \pi i k x}$. More precisely, localization (pure point spectrum with exponentially decaying eigenfunctions) results for the family $\left\{\hat{H}_{\lambda v, \alpha, \theta}\right\}_{\theta \in \mathbb{R}}$ are used to obtain information on the family $\left\{H_{\lambda v, \alpha, \theta}\right\}_{\theta \in \mathbb{R}}$. At the root of this approach is the classical Aubry duality: a Fourier-type transform matches localized eigenfunctions $\hat{H} u=E \hat{u}$ to analytic Bloch waves for the equation $H u=E u$. The duality, originally discovered in [AA], has been given several rigorous interpretations since the early 80s. A dynamical version of Aubry duality is that localization for the dual model leads to reducibility for almost every energy [P2]. A more subtle duality statement is that pure point spectrum for the dual model implies purely absolutely continuous spectrum for a.e. $\theta$ [GJLS]. However, all the duality links established so far have been entirely algebraic, with no quantitative estimates involved. Thus they could not lead to an understanding of the whole spectrum, since they ignore the set of energies where localization (and reducibility) fails. Inability to study this (zero measure yet topologically generic) set of bad energies was a major stumbling block in answering various spectral questions, including some longstanding conjectures. For example, the question whether potential gaps can collapse, whether there is ever any singular spectrum, or what is the exact modulus of continuity of the integrated density of states all require estimates holding for all energies. 
In this paper we address this issue by developing the first quantitative version of duality, which makes it possible to obtain fine dynamical estimates from local information on the dual model. We then show that required local properties do hold non-perturbatively for all energies in the spectrum, thus allowing us to obtain all of Eliasson's results (and more) in the non-perturbative setting. As discussed above, results "for almost every energy" can be obtained by considering only the localized solutions for the dual model. Our achievement here is in developing the technique that can handle all energies, including those for which localization does not hold, and there is no reducibility. For those energies, our approach still gives tight dynamical estimates that imply "almost reducibility": though coordinate changes cannot trivialize the dynamics (this would be reducibility), they come arbitrarily close to it (Theorem 1.4). Here the notion of closeness is rather strong, involving control in a fixed band of analyticity (such strong control is new even in the perturbative regime).

Besides almost reducibility, the dynamical estimates yield almost immediately a number of corollaries, including sharp estimates such as $1 / 2$-Hölder continuity of the integrated density of states (Theorems 1.2 and 1.6, and the non-collapse of spectral gaps for the almost Mathieu operator (Theorem 1.1). Certain other consequences are less immediate and will be reported separately.

Our almost reducibility result obviously implies that the non-perturbative setting can be reduced (via coordinate change) to the perturbative regime 1 so the known results in the perturbative theory become automatically non-perturbative. This is applied to deduce absolute continuity of spectral measures for all phases (Theorems 1.3 and 1.5 using a result of $[\mathrm{E}]$. While this reduction also gives other corollaries, we stress that Theorems $1.1,1.2$, and 1.6 are obtained here without using this route.

Remark 1.1. In [E], Eliasson is actually able to deal with multifrequency potentials. It was shown by Bourgain [B2] that Eliasson's results, in the multifrequency case, do not hold non-perturbatively.

Remark 1.2. A further common advantage of non-perturbative approaches, which we will not pursue here, is to extend through much weaker Diophantine conditions than what can be covered by KAM based methods, which usually stop working at the Brjuno condition. The possibility of going beyond Brjuno is very interesting for certain problems; see for instance [AJ1].

\subsection{The almost Mathieu operator}

Before stating precisely the non-perturbative version of Eliasson's result, we discuss the almost Mathieu operator. Results are particularly neat for this case because the dual model of the almost Mathieu operator is a rescaled almost Mathieu operator (with the same frequency, but inverse coupling), thus the non-perturbative analysis extends through the

\footnotetext{
1 Theorem 1.4 is actually much more than is needed for mere reduction to the perturbative regime, which in itself is a less delicate result (it is presented in the appendix as Theorem A.2.
} 
whole subcritical regime $|\lambda|<1$ (and cannot be extended further, as the almost Mathieu operator undergoes a phase transition at $|\lambda|=1[\mathrm{AA}]$ ).

In [AJ1], it was proved that the spectrum of the almost Mathieu operator is a Cantor set for any $\alpha \in \mathbb{R} \backslash \mathbb{Q}, \lambda \neq 0$. This is the Ten Martini Problem of Kac-Simon. There is a much more difficult problem, which remains open, known as the dry version of the Ten Martini Problem: it asks to show that the spectrum is not only a Cantor set, but that all gaps predicted by the Gap-Labelling Theorem [BLT, JM] are open (see Section 4.2 for a precise formulation). In contrast with the Ten Martini Problem that only requires a certain property to hold densely in the spectrum, this formulation requires handling all energies with rational rotation numbers, thus does not leave any room for energy exclusion. The dry Ten Martini Problem has enjoyed a significant attention since its first formulation in the early 80s. An affirmative answer was obtained for Liouville $\alpha$ [CEY], as well as for a set of $(\lambda, \alpha)$ of positive Lebesgue measure [P1]. Here we are able to deal with almost every $(\lambda, \alpha)$.

Let $\mathrm{DC}(\kappa, \tau)$ be the set of $\alpha \in \mathbb{R}$ such that $|\alpha-p / q| \geq \kappa q^{-\tau}, p, q \in \mathbb{Z}, q \neq 0$. The set of Diophantine numbers is $\mathrm{DC}=\bigcup_{\kappa>0, \tau>0} \mathrm{DC}(\kappa, \tau)$.

Theorem 1.1. The dry version of the Ten Martini Problem holds for $\alpha \in \mathrm{DC}, \lambda \neq$ $-1,0,1$.

The work of Puig mentioned above yields the same result in the perturbative regime $\alpha \in$ $\mathrm{DC}, \ln |\lambda|$ large (depending on $\alpha$ ), and is based on the perturbative results of Eliasson.

We note that our proof does not depend on [AJ1], where the issue was in handling the arithmetically critical non-Diophantine regime, while here we entirely focus on the Diophantine case.

As discussed in [AJ1], in order to bridge the gap between generic and full measure, one must analyze a particularly difficult region of parameters which is still badly understood (and which this work does not touch). The case $|\lambda|=1$ is also very open even in the case when $\alpha$ is the golden mean (the problem is that one cannot use localization methods).

We next move our focus to the regularity of the integrated density of states, a recurring theme in the analysis of quasiperiodic operators.

Theorem 1.2. Let $\alpha \in \mathrm{DC}, \lambda \neq-1,0,1$. Then the integrated density of states is $1 / 2$ Hölder continuous.

This estimate is optimal in several ways. First, there are square-root singularities at the boundaries of gaps (e.g., [P2]), so the modulus of continuity cannot be improved. Also, it is known that for a certain non-empty set of $\alpha$ with good Diophantine properties (but of zero Lebesgue measure) and $\lambda=1$, the integrated density of states is not Hölder ([B3, Remark after Corollary 8.6]). Finally, for any $\lambda \neq 0$ and generic $\alpha$, the integrated density of states is not Hölder (this is because the Lyapunov exponent is discontinuous at rational $\alpha$, which easily implies that it is not Hölder for generic $\alpha$ ).

Goldstein-Schlag [GS] had previously shown $(1 / 2-\epsilon)$-Hölder continuity for any $\epsilon$, all $\lambda \neq-1,0,1$ and a full Lebesgue measure subset of Diophantine frequencies (they are 
also able to consider other analytic functions, in the regime of positive Lyapunov exponent, at the cost of a worse Hölder constant). Previously Bourgain [B1] had obtained almost 1/2-Hölder continuity in the perturbative regime, for Diophantine $\alpha$ and $\ln |\lambda|$ large (depending on $\alpha$ ). More recently Sana Ben Hadj Amor obtained 1/2-Hölder continuity in the perturbative regime of Eliasson $\mathrm{Am}$.

Another consequence of our results is the following. A well known conjecture, dating back to the work of Aubry-André [AA], and more recently included in the list of problems of Simon (Problem 6 of [S2]), asks to show that for $0<|\lambda|<1$ the spectral measures of the almost Mathieu operator are absolutely continuous. This is also tied to another, more general and far-reaching, conjecture, sometimes attributed to Simon, that for almost periodic operators, singular spectrum must be phase independent. While a.e. phase-independence of the spectrum and of its a.c., s.c. and pure point components is an almost immediate corollary of ergodicity, almost periodic operators exhibit a certain phase rigidity, in that the spectrum [AS] and absolutely continuous spectrum [LS] are the same for all phases. Although this is not true for singular continuous and pure point components taken individually (there may be a dependence on the arithmetic of the phase [JS]), the conjecture is that combined together they will also demonstrate the phase stability. This question is only non-trivial (but highly so) in the regime of zero Lyapunov exponents. We should note that by the time of the inclusion of the above question as Problem 6 in [S2], it was already solved in the case of Diophantine frequencies for a.e. phase $[\bar{J}]$, thus, in the Diophantine regime, the issue was precisely the phase rigidity. This is what we address here.

Theorem 1.3. The spectral measures of the almost Mathieu operator are absolutely continuous for $\alpha \in \mathrm{DC}, 0<|\lambda|<1, \theta \in \mathbb{R}$.

In the perturbative regime, $\alpha \in \mathrm{DC}, 0<|\lambda|<\lambda_{0}(\alpha)$, the result for all $\theta \in \mathbb{R}$ follows from the work of Eliasson. As already mentioned, Theorem 1.3 is obtained here from Eliasson's result by "non-perturbative reduction to the perturbative regime".

Remark 1.3. Though this is not relevant to this work (since the estimates involved in our reduction to the perturbative regime are already bound to the usual Diophantine condition), we should point out that this approach does limit possible extensions beyond the Brjuno condition (see Remark 1.2). Such issues are particularly relevant in view of recent progress towards absolutely continuous spectrum "from the Liouville side" [AD], which seems to break down strictly beyond the Brjuno condition 2 while absolutely continuous spectrum is expected to hold, as described above, without any exceptions 3

\footnotetext{
2 Namely, the method of $[\mathrm{AD}$ can only cover irrational numbers that are exponentially well approximated by rationals.

3 After this work was completed, the first author has obtained a complete solution to Problem 6 of [S2], which does involve the development of a fully non-perturbative approach to absolutely continuous spectrum.
} 


\subsection{Almost reducibility}

As previously discussed, Eliasson's analysis is based on dynamical systems considerations. A cocycle is defined by a pair $(\alpha, A)$ where $\alpha \in \mathbb{R}$ and $A: \mathbb{R} / \mathbb{Z} \rightarrow \operatorname{SL}(2, \mathbb{R})$ is analytic. It is viewed as a linear skew-product $(x, w) \mapsto(x+\alpha, A(x) \cdot w), x \in \mathbb{R} / \mathbb{Z}$, $w \in \mathbb{R}^{2}$. We say that two analytic cocycles $\left(\alpha, A^{(i)}\right), i=1,2$, are analytically conjugate if there exists an analytic map $B: \mathbb{R} / \mathbb{Z} \rightarrow \operatorname{PSL}(2, \mathbb{R})$ such that

$$
A^{(2)}(x)=B(x+\alpha) A^{(1)}(x) B(x)^{-1} .
$$

The dynamical properties of cocycles are preserved by conjugacies. We say that a cocycle is reducible if it is $C^{\omega}$-conjugate to a cocycle of the form $\left(\alpha, A_{*}\right)$ where $A_{*}$ is a constant matrix. Eliasson's reducibility theory describes the dynamics of $(\alpha, A)$ when $\alpha$ is Diophantine and $A$ is close to a constant. He shows that such cocycles are typically (in a measure-theoretic sense) reducible, and gives good estimates for the non-reducible ones. The precise closeness quantifier defines Eliasson's perturbative regime. See the appendix for a summary of the results of the theory that are relevant to this work.

Eliasson's perturbative regime is not invariant under conjugacies. A more intrinsic notion, almost reducibility, introduced by Avila-Krikorian $[\mathrm{AK} 2]^{4}$ in the smooth category, captures the properties of cocycles "that behave as cocycles in Eliasson's perturbative regime". The results of this work justify introducing the corresponding definition in the analytic category as well.

Definition 1.1. An analytic cocycle $(\alpha, A)$ is $C^{\omega}$-almost reducible if the closure of its analytic conjugacy class contains a constant.

If $(\alpha, A)$ is $C^{\omega}$-almost reducible and $\alpha$ is Diophantine, then $(\alpha, A)$ is analytically conjugate to a cocycle in Eliasson's perturbative regime.

The connection to Schrödinger operators is clear when

$$
A(x)=S_{\lambda v, E}(x)=\left(\begin{array}{cc}
E-\lambda v(x) & -1 \\
1 & 0
\end{array}\right),
$$

since a solution of $H u=E u$ satisfies

$$
A(\theta+n \alpha)\left(\begin{array}{c}
u_{n} \\
u_{n-1}
\end{array}\right)=\left(\begin{array}{c}
u_{n+1} \\
u_{n}
\end{array}\right) .
$$

In other words, the spectral properties of the family of Schrödinger operators $\left\{H_{\lambda v, \alpha, \theta}\right\}_{\theta \in \mathbb{R}}$ are closely related to the dynamics of the family of cocycles $\left\{\left(\alpha, S_{\lambda v, E}\right)\right\}_{E \in \mathbb{R}}$.

Theorem 1.4. For $\alpha \in \mathrm{DC}, v: \mathbb{R} / \mathbb{Z} \rightarrow \mathbb{R}$ analytic, and $0<|\lambda|<\lambda_{0}(v)$, the cocycles associated with $\left\{H_{\lambda v, \alpha, \theta}\right\}_{\theta \in \mathbb{R}}$ are almost reducible. If $v(x)=2 \cos 2 \pi x$ then $\lambda_{0}=1$.

Direct application of Eliasson's reducibility theory immediately yields a generalized version of Theorem 1.3 .

\footnotetext{
4 See also the first (preprint) version of [AK1].
} 
Theorem 1.5. Let $\alpha, v, \lambda$ be as in Theorem 1.4 Then the singular spectrum is the same for all phases $\theta \in \mathbb{R}$ (and empty).

We note that in the non-perturbative regime, the a.e. absolutely continuous spectrum was established in [BJ1]. What we address here is the stability of singular spectrum.

\subsection{Further non-perturbative analysis}

Theorem 1.6. Let $\alpha, v, \lambda$ be as in Theorem 1.4 Then the integrated density of states is 1/2-Hölder.

The history of this question is discussed after Theorem 1.2 , and as for the almost Mathieu case, this result is optimal in several ways. While we give a direct simple proof, this theorem can also be derived via Theorem 1.4 from a recent perturbative result of Sana Ben Hadj Amor [Am].

Our analysis also allows studying a more delicate question: Hölder continuity of the individual spectral measures (of which the integrated density of states is an average). We can show that for all $\theta$ and all localized initial vectors their spectral measures are uniformly $1 / 2$-Hölder. This result is once again optimal, and new even in the perturbative regime. This corollary is a little more involved and also needs the introduction of some additional theory. It will be reported separately [AJ2].

The dry version of the Ten Martini Problem is specific to the almost Mathieu operator, and does not hold for general analytic potentials [DJ]. What can be concluded is that for the great majority of $v$ (that is, excluding a set of infinite codimension), and except for countably many $0<|\lambda|<\lambda_{0}(v)$, all gaps are open.

\subsection{Almost localization and quantitative Aubry duality}

Our key problem is thus to show almost reducibility of certain cocycles. As mentioned before, there is a classical Aubry duality link between localization and reducibility (Theorem 2.5), whose application is however limited since localization (pure point spectum with exponentially decaying eigenfunctions) in general does not hold for every $\theta$ [JS]. This of course fits with the fact that reducibility in general does not hold for all cocycles. (Localization for almost every $\theta$, proved in [J] and [BJ1], turns out to be enough for many results, but not for the fine ones we are interested in here.)

It is thus natural to devise a weakened notion of localization that could be expected to hold for every phase, and to develop ways to link it to almost reducibility. Here we show that this approach indeed works. Namely, we establish a quantitative version of Aubry duality that links local exponential decay of solutions to the dual eigenvalue problem to fine dynamical estimates, thus showing that almost reducibility of cocycles associated with $\left\{H_{\lambda v, \alpha, \theta}\right\}_{\theta \in \mathbb{R}}$ can be deduced from a property of the dual model $\left\{\hat{H}_{\lambda v, \alpha, \theta}\right\}$, which we call "almost localization" (see Definition 3.1. Informally, almost localization gives 
a precise description of the decay of generalized eigenfunctions away from a sparse sequence of resonances, somewhat similar to what is considered in [JL], but more precise than what is obtained in [J] and [BJ1]. Refining those results, we indeed show in Theorem 3.2 that almost localization holds for the dual model in the regime relevant for Theorem 1.4. Then we proceed to show that for $\alpha \in \mathrm{DC}$, almost localization for the dual model implies almost reducibility. This link is quantitative and is significantly more subtle than that between reducibility and localization. The key dynamical estimates needed to establish this link, Theorems 3.4 and 3.5. coupled with Theorem 3.2 immediately imply Theorem 1.4 (and all its corollaries). They also directly imply Theorems 1.1, 1.2, 1.6, Those direct implications are not difficult but involve conceptually new arguments, presented in $\$ 4$ Particularly, the direct proof of Theorem 1.1 is based on linking resonant rotation numbers to resonant phases (see Theorem 4.2 or Theorem 4.1 for more detail). We note that the proof of Theorem 3.2 (\$5) builds on well developed localization methods, and borrows a number of ingredients from [BJ1] and some shortcuts from [AJ1]. Theorems 3.4 and 3.5 and their proofs, presented in Sections 6, 7, and 8, are the main novel technical and conceptual contributions of this paper, and are not based on previous work.

Remark 1.4. Previous progress in extending Eliasson's results by coupling non-perturbative and perturbative methods was obtained by Avila-Krikorian. In [AK1], almost every energy results were obtained following a rather different technique (reduction to the perturbative regime was achieved by renormalization). More precise (unpublished) results were later obtained (using the connection between localization and reducibility in both directions), including a non-perturbative proof of reducibility under a topological condition (which covered "most" of the spectrum but excluded the gap boundaries).

Let us conclude with a few comments on the analysis of general operators $(1.1)$, without restriction on the coupling constant ${ }^{5}$ There has been much recent progress on the description of the part of the spectrum $\Sigma_{+}$corresponding to energies with a positive Lyapunov exponent (see [B3] for a lengthy account, and [GS] for more recent results). The concept of almost reducibility allows us to determine another region of the spectrum $\Sigma_{a r}$ which can be thoroughly analyzed (either directly by the methods developed here, or by reduction to the perturbative regime).

There are a number of parallels between our work and previous developments regarding the positive Lyapunov exponent regime. For instance, the stability of positivity of the Lyapunov exponents (in other words, the property that the set of energies corresponding to zero Lyapunov exponent is closed) [BJ2] is paralleled by the fact that it is also possible to deduce stability of almost reducibility from Theorem 1.4 , utilizing an argument of [AK2]. It is precisely the stability of almost reducibility that makes the concept so interesting from the dynamical systems point of view.

It is interesting to note that almost reducibility may be after all determined by a "mirror" condition to positivity of the Lyapunov exponent. Indeed, for energies in the spectrum, almost reducibility clearly implies strong vanishing of the Lyapunov exponent, in

\footnotetext{
5 Earlier, less complete (due to reliance on perturbative techniques) considerations (in the case of smooth potentials) first appeared in the preprint version of [AK1].
} 
the sense that the cocycle may grow at most subexponentially in some band. We conjecture that the converse also holds, that is, almost reducibility should follow from strong vanishing of the Lyapunov exponent.

\section{Preliminaries}

For a bounded analytic function $f$ defined on a strip $\{|\mathfrak{s} z|<\epsilon\}$ we let $\|f\|_{\epsilon}=$ $\sup _{|\Im z|<\epsilon}|f(z)|$. If $f$ is a bounded continuous function on $\mathbb{R}$, we define $\|f\|_{0}=$ $\sup _{x \in \mathbb{R}}|f(x)|$.

\subsection{Cocycles}

Let $\alpha \in \mathbb{R} \backslash \mathbb{Q}$ and $A \in C^{0}(\mathbb{R} / \mathbb{Z}, \operatorname{SL}(2, \mathbb{C}))$. We call $(\alpha, A)$ a (complex) cocycle. The Lyapunov exponent is given by the formula

$$
L(\alpha, A)=\lim _{n \rightarrow \infty} \frac{1}{n} \int \ln \left\|A_{n}(x)\right\| d x,
$$

where $A_{n}, n \in \mathbb{Z}$, is defined by $(\alpha, A)^{n}=\left(n \alpha, A_{n}\right)$, so that for $n \geq 0$,

$$
A_{n}(x)=A(x+(n-1) \alpha) \cdots A(x) .
$$

It turns out (since irrational rotations are uniquely ergodic) that

$$
L(\alpha, A)=\lim _{n \rightarrow \infty} \sup _{x \in \mathbb{R} / \mathbb{Z}} \frac{1}{n} \ln \left\|A_{n}(x)\right\| .
$$

Remark 2.1. By subadditivity, for any compact set $K \subset(\mathbb{R} \backslash \mathbb{Q}) \times C^{0}(\mathbb{R} / \mathbb{Z}, \operatorname{SL}(2, \mathbb{R}))$, for every $\delta>0$ there exists $C_{K, \delta}>0$ such that for every $k \geq 0$,

$$
\sup _{(\alpha, A) \in K} \sup _{x \in \mathbb{R} / \mathbb{Z}} \ln \left\|A_{k}(x)\right\| \leq C_{K, \delta}+k\left(\sup _{(\alpha, A) \in K} L(\alpha, A)+\delta\right) .
$$

We say that $(\alpha, A)$ is uniformly hyperbolic if there exists a continuous splitting $\mathbb{C}^{2}=$ $E^{s}(x) \oplus E^{u}(x), x \in \mathbb{R} / \mathbb{Z}$, such that for some $C, c>0$, and for every $n \geq 0,\left\|A_{n}(x) \cdot w\right\| \leq$ $C e^{-c n}\|w\|$ for $w \in E^{s}(x)$ and $\left\|A_{-n}(x) \cdot w\right\| \leq C e^{-c n}\|w\|$ for $w \in E^{u}(x)$. In this case, of course $L(\alpha, A)>0$. We say that $(\alpha, A)$ is bounded if $\sup _{n \geq 0} \sup _{x \in \mathbb{R} / \mathbb{Z}}\left\|A_{n}(x)\right\|<\infty$.

Given two cocycles $\left(\alpha, A^{(1)}\right)$ and $\left(\alpha, A^{(2)}\right)$, a (complex) conjugacy between them is a continuous $B: \mathbb{R} / \mathbb{Z} \rightarrow \operatorname{SL}(2, \mathbb{C})$ such that 1.3 holds. The Lyapunov exponent is clearly invariant under conjugacies.

We assume now that $(\alpha, A)$ is a real cocycle, that is, $A \in C^{0}(\mathbb{R} / \mathbb{Z}, \operatorname{SL}(2, \mathbb{R}))$. The notion of real conjugacy (between real cocycles) is the same as before, except that we look for $B \in C^{0}(\mathbb{R} / \mathbb{Z}, \operatorname{PSL}(2, \mathbb{R}))$. Real conjugacies still preserve the Lyapunov exponent. An example where it is useful to allow $B: \mathbb{R} / \mathbb{Z} \rightarrow \operatorname{PSL}(2, \mathbb{R})$ instead of requiring $B: \mathbb{R} / \mathbb{Z} \rightarrow \operatorname{SL}(2, \mathbb{R})$ is given by the following well known result (see [MP] for the case of continuous time). 
Theorem 2.1. Let $(\alpha, A)$ be a uniformly hyperbolic cocycle, with $\alpha$ Diophantine and $A$ analytic. Then there exists an analytic $B: \mathbb{R} / \mathbb{Z} \rightarrow \operatorname{PSL}(2, \mathbb{R})$ such that the matrix $B(x+\alpha) A(x) B(x)^{-1}$ is constant.

One cannot always take $B: \mathbb{R} / \mathbb{Z} \rightarrow \operatorname{SL}(2, \mathbb{R})$ in Theorem $2.1^{6}$

We say that $(\alpha, A)$ is (analytically) reducible if it is (real) conjugate to a constant cocycle, and the conjugacy is analytic.

The fundamental group of $\operatorname{SL}(2, \mathbb{R})$ is isomorphic to $\mathbb{Z}$. Let

$$
R_{\theta}=\left(\begin{array}{cc}
\cos 2 \pi \theta & -\sin 2 \pi \theta \\
\sin 2 \pi \theta & \cos 2 \pi \theta
\end{array}\right)
$$

Any $A: \mathbb{R} / \mathbb{Z} \rightarrow \operatorname{SL}(2, \mathbb{R})$ is homotopic to $x \mapsto R_{n x}$ for some $n \in \mathbb{Z}$ called the degree of $A$ and denoted $\operatorname{deg} A=n$.

Assume now that $A: \mathbb{R} / \mathbb{Z} \rightarrow \operatorname{SL}(2, \mathbb{R})$ is homotopic to the identity. Then there exist $\psi: \mathbb{R} / \mathbb{Z} \times \mathbb{R} / \mathbb{Z} \rightarrow \mathbb{R}$ and $u: \mathbb{R} / \mathbb{Z} \times \mathbb{R} / \mathbb{Z} \rightarrow \mathbb{R}^{+}$such that

$$
A(x) \cdot\left(\begin{array}{c}
\cos 2 \pi y \\
\sin 2 \pi y
\end{array}\right)=u(x, y)\left(\begin{array}{c}
\cos 2 \pi(y+\psi(x, y)) \\
\sin 2 \pi(y+\psi(x, y))
\end{array}\right) .
$$

The function $\psi$ is called a lift of $A$. Let $\mu$ be any probability on $\mathbb{R} / \mathbb{Z} \times \mathbb{R} / \mathbb{Z}$ which is invariant under the continuous map $T:(x, y) \mapsto(x+\alpha, y+\psi(x, y))$, projecting over Lebesgue measure on the first coordinate (for instance, take for $\mu$ any accumulation point of $n^{-1} \sum_{k=0}^{n-1} T_{*}^{k} v$ where $v$ is Lebesgue measure on $\mathbb{R} / \mathbb{Z} \times \mathbb{R} / \mathbb{Z}$ ). Then the number

$$
\rho(\alpha, A)=\int \psi d \mu \bmod \mathbb{Z}
$$

does not depend on the choices of $\psi$ and $\mu$, and is called the fibered rotation number of $(\alpha, A)$ (see $[\mathrm{JM}]$ and $[\mathrm{H}])$.

It is immediate from the definition that

$$
|\rho(\alpha, B)-\theta|<C\left\|B-R_{\theta}\right\|_{0} .
$$

The fibered rotation number is invariant under real conjugacies which are homotopic to the identity. In general, if the cocycles $\left(\alpha, A^{(1)}\right)$ and $\left(\alpha, A^{(2)}\right)$ are real conjugate, $B(x+\alpha) A^{(2)}(x) B(x)^{-1}=A^{(1)}(x)$, and $B: \mathbb{R} / \mathbb{Z} \rightarrow \operatorname{PSL}(2, \mathbb{R})$ has degree $k$ (that is, it is homotopic to $\left.x \mapsto R_{k x / 2}\right)$ then

$$
\rho\left(\alpha, A^{(1)}\right)=\rho\left(\alpha, A^{(2)}\right)+k \alpha / 2 .
$$

For uniformly hyperbolic cocycles there is the following well known result (for $\alpha$ Diophantine and $A$ analytic, it is a consequence of Theorem 2.1 ; see $\$ 5.17$ of $[\mathrm{H}]$.

Theorem 2.2. Let $(\alpha, A)$ be a uniformly hyperbolic cocycle with $\alpha \in \mathbb{R} \backslash \mathbb{Q}$. Then $2 \rho(\alpha, A) \in \alpha \mathbb{Z}+\mathbb{Z}$.

\footnotetext{
${ }^{6}$ Indeed, the continuous splitting of $\mathbb{R}^{2}$ associated to a real uniformly hyperbolic cocycle may be topologically non-trivial (see $\S 4.3$ of $[\mathrm{H}]$ for an example).
} 


\subsection{Schrödinger operators}

We now consider Schrödinger operators $\left\{H_{v, \alpha, \theta}\right\}_{\theta \in \mathbb{R}}$ (we incorporate the coupling constant into $v$ ). The spectrum $\Sigma=\Sigma_{v, \alpha}$ does not depend on $\theta$, and it is the set of $E$ such that $\left(\alpha, S_{v, E}\right)$ is not uniformly hyperbolic, with $S_{v, E}$ given by (1.4).

The Lyapunov exponent is defined by $L(E)=L\left(\alpha, S_{v, E}\right)$.

Fixing a phase $\theta$ and $f \in l^{2}(\mathbb{Z})$, we let $\mu=\mu_{v, \alpha, \theta}^{f}$ be the spectral measure of $H=H_{v, \alpha, \theta}$ corresponding to $f$. It is defined so that

$$
\left\langle(H-E)^{-1} f, f\right\rangle=\int_{\mathbb{R}} \frac{1}{E^{\prime}-E} d \mu\left(E^{\prime}\right)
$$

for $E$ in the resolvent set $\mathbb{C} \backslash \Sigma$.

The integrated density of states is the function $N: \mathbb{R} \rightarrow[0,1]$ defined by

$$
N(E)=\int_{\mathbb{R} / \mathbb{Z}} \mu_{v, \alpha, \theta}^{f}(-\infty, E] d \theta,
$$

where $f \in l^{2}(\mathbb{Z})$ is such that $\|f\|_{l^{2}(\mathbb{Z})}=1$ (the definition is independent of the choice of $f$ ). It is a continuous non-decreasing surjective (for bounded potentials) function. The Thouless formula relates the Lyapunov exponent to the integrated density of states:

$$
L(E)=\int_{\mathbb{R}} \ln \left|E^{\prime}-E\right| d N\left(E^{\prime}\right) .
$$

There is also a relation to the fibered rotation number:

$$
N(E)=1-2 \rho\left(\alpha, S_{v, E}\right)
$$

where $\rho\left(\alpha, S_{v, E}\right) \in[0,1 / 2]$.

\subsection{The dual model}

It turns out that the spectrum $\hat{\Sigma}$ of $\hat{H}=\hat{H}_{v, \alpha, \theta}$ coincides with the spectrum $\Sigma$ of $H=$ $H_{v, \alpha, \theta}$, a manifestation of Aubry duality (e.g., [GJLS]). The spectral measures $\hat{\mu}=\hat{\mu}_{v, \alpha, \theta}^{f}$ can be defined using the analogous formula to $(2.10)$. The integrated density of states $\hat{N}(E)=\int \hat{\mu}_{\theta}(-\infty, E] d \theta$ then coincides with $N(E)$. Berezanskiiı's theorem [Be, S1] gives in this context:

Theorem 2.3. For any $v, \alpha, \theta, f$, and $\hat{\mu}_{v, \alpha, \theta^{-}}^{f}$ almost every E, there exists a non-zero solution of $\hat{H} \hat{u}=E \hat{u}$ with $\left|\hat{u}_{k}\right| \leq 1+|k|$.

Since $\Sigma=\hat{\Sigma}$ is the union of the supports of the spectral measures, this implies:

Theorem 2.4. For any $v, \alpha, \theta$, there exists a dense set of $E \in \Sigma=\Sigma_{v, \alpha}$ such that there exists a non-zero solution of $\hat{H} \hat{u}=E \hat{u}$ with $\left|\hat{u}_{k}\right| \leq 1+|k|$. 


\subsection{Localization and reducibility: dynamical formulation of Aubry duality}

We describe the connection between localization and reducibility mentioned in the introduction. The theorem below is essentially a careful dynamical formulation of the classical Aubry duality. It appears in a similar form in [P2].

Theorem 2.5. Let $\alpha \in \mathbb{R} \backslash \mathbb{Q}$ and let $v: \mathbb{R} / \mathbb{Z} \rightarrow \mathbb{R}$ be analytic. Let $\theta, E \in \mathbb{R}$ be such that there exists a non-zero exponentially decaying solution of $\hat{H} \hat{u}=E \hat{u}$, and let $A=S_{v, E}$.

(1) If $2 \theta \notin \alpha \mathbb{Z}+\mathbb{Z}$, then there exists $B: \mathbb{R} / \mathbb{Z} \rightarrow \operatorname{SL}(2, \mathbb{R})$ analytic such that $B(x+\alpha) A(x) B(x)^{-1}=R_{ \pm \theta}\left(\right.$ so $(\alpha, A)$ is reducible). In particular, $\left\|A_{n}(x)\right\|=O(1)$, $x \in \mathbb{R} / \mathbb{Z}$.

(2) If $2 \theta-k \alpha \in \mathbb{Z}$ for some $k \in \mathbb{Z}$, then there exists $B: \mathbb{R} / \mathbb{Z} \rightarrow \operatorname{PSL}(2, \mathbb{R})$ and $\kappa: \mathbb{R} / \mathbb{Z} \rightarrow \mathbb{R}$ analytic such that

$$
B(x+\alpha) A(x) B(x)^{-1}=\left(\begin{array}{cc} 
\pm 1 & \kappa(x) \\
0 & \pm 1
\end{array}\right) .
$$

In particular, $\left\|A_{n}(x)\right\|=O(n), x \in \mathbb{R} / \mathbb{Z}$. If moreover $\alpha \in \mathrm{DC}$, then $\kappa$ can be chosen to be a constant (and $(\alpha, A)$ is reducible).

In either case $\rho\left(\alpha, S_{v, E}\right)= \pm \theta+m \alpha / 2$ for some $m \in \mathbb{Z}$.

Proof. Let $u(x)=\sum \hat{u}_{k} e^{2 \pi i k x}$ and $U(x)=\left(\begin{array}{c}e^{2 \pi i \theta} u(x) \\ u(x-\alpha)\end{array}\right)$. Then we have $A(x) \cdot U(x)=$ $e^{2 \pi i \theta} U(x+\alpha)$. Let $\tilde{B}(x)$ be the matrix with columns $U(x)$ and $\bar{U}(x)$. By minimality of $x \mapsto x+\alpha, \operatorname{det} \tilde{B}$ is a constant.

(Case A) If det $\tilde{B} \neq 0$, we have $\tilde{B}(x+\alpha)^{-1} A(x) \tilde{B}(x)=\left(\begin{array}{cc}e^{2 \pi i \theta} & 0 \\ 0 & e^{-2 \pi i \theta}\end{array}\right)$. It is easy to see that $\operatorname{det} \tilde{B}= \pm c i$ for some $c>0$; we then take $B^{-1}=(2 c)^{-1 / 2} \tilde{B}\left(\begin{array}{c}1 \\ 1 \neq i \\ \mp i\end{array}\right)$.

(Case B) If $\operatorname{det} \tilde{B}=0$ then $U(x)=\psi(x) W(x)$ with $W(x)$ a real vector defined up to sign and $|\psi(x)|=1$. By minimality of $x \mapsto x+\alpha, W(x) \neq 0$ for every $x \in \mathbb{R} / \mathbb{Z}$. The matrix $B(x)^{-1} \in \operatorname{PSL}(2, \mathbb{R})$ with columns $W$ and $\|W(x)\|^{-2} R_{1 / 4} W(x)$ is thus well defined. Then

$$
B(x+\alpha) A(x) B(x)^{-1}=\left(\begin{array}{cc}
d(x) & \kappa(x) \\
0 & d(x)^{-1}
\end{array}\right), \quad \text { where } \quad d(x)=\frac{\psi(x+\alpha)}{\psi(x)} e^{2 \pi i \theta} .
$$

Since $|d(x)|=1$ and $d(x)$ is real, $d(x)= \pm 1$. If $\alpha \in \mathrm{DC}$, we can also further conjugate $A$ to a constant parabolic (or identity) matrix by solving (using, say, Fourier series) the cohomological equation $\pm \phi(x+\alpha) \mp \phi(x)=\kappa(x)-\int_{0}^{1} \kappa(x) d x$ with $\int_{0}^{1} \phi(x) d x=0$ in $\mathbb{R} / \mathbb{Z}$. Letting $B^{\prime}(x)=\left(\begin{array}{cc}1 & -\phi(x) \\ 0 & 1\end{array}\right) B(x)$, we get

$$
B^{\prime}(x+\alpha) A(x) B^{\prime}(x)^{-1}=\left(\begin{array}{cc} 
\pm 1 & \int_{0}^{1} \kappa(x) d x \\
0 & \pm 1
\end{array}\right) .
$$

Assume $2 \theta \notin \alpha \mathbb{Z}+\mathbb{Z}$. Then we cannot be in case B: indeed, $e^{2 \pi i \theta}= \pm \psi(x) / \psi(x+\alpha)$ implies (using Fourier series) that $\psi(x)=e^{-\pi i k x}$ (notice that $\psi$ is well defined only in 
$\mathbb{R} / 2 \mathbb{Z})$ and $e^{2 \pi i \theta}= \pm e^{\pi i k \alpha}$, that is, $2 \theta=k \alpha \bmod \mathbb{Z}$. Thus we are in case $\mathrm{A}$, and the first statement follows immediately.

Assume now that $2 \theta-k \alpha \in \mathbb{Z}$. If we are in case $\mathrm{B}$, then the second statement follows immediately. Otherwise we are in case $\mathrm{A}$, and we have $B(x+\alpha) A(x) B(x)^{-1}=$ $R_{ \pm \theta}$ for some $B: \mathbb{R} / \mathbb{Z} \rightarrow \operatorname{SL}(2, \mathbb{R})$. We then set $B^{\prime}(x)=R_{\mp k x / 2} B(x)$, and we get $B^{\prime}(x+\alpha) A(x) B^{\prime}(x)^{-1}= \pm$ id. So the second statement still follows in this case (with $\kappa(x)=0)$.

The statement about the value of $\rho$ follows immediately from 2.9.

If $2 \theta \in \alpha \mathbb{Z}+\mathbb{Z}$, we will say that $\theta$ is rational (with respect to $\alpha$ ).

Remark 2.2. It is clear from the above proof that if the Fourier transform of $\hat{u}$ is analytic in a strip $|\Im z|<\epsilon$ then the matrix $B$ given in item (1) is analytic in the same strip. As for item (2), it is still possible to define a conjugating matrix with a definite complex extension, but one must be more careful in its definition.

\subsection{Rational approximations}

Let $q_{n}$ be the denominators of the approximants of $\alpha$. We recall the basic properties:

$$
\begin{gathered}
\left\|q_{n} \alpha\right\|_{\mathbb{R} / \mathbb{Z}}=\inf _{1 \leq k \leq q_{n+1}-1}\|k \alpha\|_{\mathbb{R} / \mathbb{Z}} \\
1 \geq q_{n+1}\left\|q_{n} \alpha\right\|_{\mathbb{R} / \mathbb{Z}} \geq 1 / 2
\end{gathered}
$$

One aspect of the "good distribution" of orbits $\{x+j \alpha\}_{j=0}^{q_{n}-1}$ which will find repeated use in this work is the following estimate.

Lemma 2.6 (Lemma 9.7 of $[\mathrm{AJ} 1]$ ). Let $\alpha \in \mathbb{R} \backslash \mathbb{Q}, x \in \mathbb{R}$ and let $0 \leq l_{0} \leq q_{n}-1$ be such that $\left|\sin \pi\left(x+l_{0} \alpha\right)\right|$ is minimal. Then for some absolute constant $C>0$,

$$
-C \ln q_{n} \leq \sum_{\substack{l=0 \\ l \neq l_{0}}}^{q_{n}-1} \ln |\sin \pi(x+l \alpha)|+\left(q_{n}-1\right) \ln 2 \leq C \ln q_{n} .
$$

\section{General setup and statements of the main (localization and dynamical) estimates}

\subsection{Almost localization for every $\theta$}

Let $\alpha \in \mathbb{R}, \theta \in \mathbb{R}, \epsilon_{0}>0$. We say that $k$ is an $\epsilon_{0}$-resonance if $\|2 \theta-k \alpha\|_{\mathbb{R} / \mathbb{Z}} \leq e^{-|k| \epsilon_{0}}$

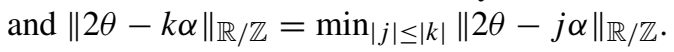

Remark 3.1. In particular, there always exists at least one resonance, 0 . If $\alpha \in \operatorname{DC}(\kappa, \tau)$,

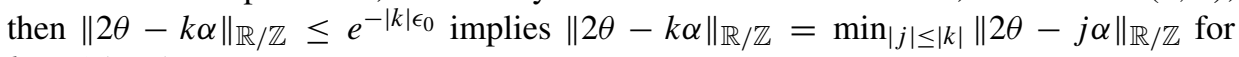
$k>C(\kappa, \tau)$. 
We order the $\epsilon_{0}$-resonances as $0=n_{0}<\left|n_{1}\right| \leq\left|n_{2}\right| \leq \cdots$. We say that $\theta$ is $\epsilon_{0^{-}}$ resonant if the set of resonances is infinite. If $\theta$ is non-resonant, with the set of resonances $\left\{n_{0}, \ldots, n_{j}\right\}$, we formally set $n_{j+1}=\infty$. The Diophantine condition immediately implies exponential repulsion of resonances:

Lemma 3.1. If $\alpha \in \mathrm{DC}(\kappa, \tau)$, then $\left|n_{j+1}\right| \geq c\left\|2 \theta-n_{j} \alpha\right\|_{\mathbb{R} / \mathbb{Z}}^{-c} \geq c e^{c \epsilon_{0}\left|n_{j}\right|}$, where $c=$ $c\left(\kappa, \tau, \epsilon_{0}\right)>0$.

Remark 3.2. In case $\left\|2 \theta-\ell_{0} \alpha\right\|_{\mathbb{R} / \mathbb{Z}}=0$ (so that $\theta$ is rational with respect to $\alpha$ ) we have an especially strong resonance at $\ell_{0}$. In particular, $\theta$ is non-resonant, as there will be no resonances $n$ with $|n|>\left|\ell_{0}\right|$.

A simple Borel-Cantelli argument shows that the set of non-resonant $\theta$ has full Lebesgue measure.

Definition 3.1. We say that the family $\left\{\hat{H}_{v, \alpha, \theta}\right\}_{\theta \in \mathbb{R}}$ exhibits almost localization if there exist $C_{0}, C_{1}, \epsilon_{0}, \epsilon_{1}>0$ such that for every solution $\hat{u}$ of $\hat{H}_{v, \alpha, \theta} \hat{u}=$ E $\hat{u}$ satisfying $\hat{u}_{0}=1$ and $\left|\hat{u}_{k}\right| \leq 1+|k|$, and for every $C_{0}\left(1+\left|n_{j}\right|\right)<k<C_{0}^{-1}\left|n_{j+1}\right|$, we have $\left|\hat{u}_{k}\right| \leq C_{1} e^{-\epsilon_{1} k}$ (where the $n_{j}$ are the $\epsilon_{0}$-resonances of $\theta$ ).

Remark 3.3. It is clear from Theorem 2.3 that almost localization implies localization for non-resonant $\theta$ (slowly growing generalized eigenfunctions can always be shifted and normalized to match the definition).

Theorem 3.2. If $v: \mathbb{R} / \mathbb{Z} \rightarrow \mathbb{R}$ is analytic and $7|\lambda|<\lambda_{0}(v)$ then $\left\{\hat{H}_{\lambda v, \alpha, \theta}\right\}_{\theta \in \mathbb{R}}$ is almost localized for every $\alpha \in$ DC. For $v(x)=2 \cos 2 \pi x$, we have $\lambda_{0}=1$.

This theorem will be proved in $\$ 5$

\subsection{Bounded eigenfunctions for every energy}

The next result allows one to pass from "every $\theta$ " statements to "every $E$ " statements.

Theorem 3.3. If $E \in \Sigma$ then there exists $\theta \in \mathbb{R}$ and a bounded solution of $\hat{H}_{v, \alpha, \theta} \hat{u}=E \hat{u}$ with $\hat{u}_{0}=1$ and $\left|\hat{u}_{n}\right| \leq 1$.

Proof. It is enough to show this for a dense set of $E$ in the spectrum. By Theorem 2.4 we may assume that there is a generalized eigenfunction $\hat{u}^{\prime}$ with subexponential growth for some phase. Fix $N>0, \epsilon>0$. Let $k_{i}$ be a sequence such that $\left|\hat{u}_{k_{i}}^{\prime}\right|=\max _{|j| \leq(i+1) N}\left|\hat{u}_{j}^{\prime}\right|$. It follows that there exist some (and indeed infinitely many) $i$ with $\left|\hat{u}_{k_{i+1}}^{\prime}\right| \leq(1+\epsilon)\left|\hat{u}_{k_{i}}^{\prime}\right|$. By shifting the phase and rescaling, we obtain, for every $\epsilon>0, N>0$, some phase $\theta^{N, \epsilon}$

7 The proof actually gives a quantitative bound on the dependence of $\lambda_{0}$ on the analytic extension of $v$. More precisely, what is needed is that $\sup _{|\Im x|<\epsilon}\left|\lambda_{0} v(x)\right| \leq c_{0} \epsilon^{k_{0}}$ for some $0<\epsilon<1$, where $c_{0}$ and $k_{0}$ are absolute constants (see [5.15), (5.19), (5.20). 
such that there exists an eigenfunction with $\hat{u}_{0}^{N, \epsilon}=1$ and $\left|\hat{u}_{n}^{N, \epsilon}\right| \leq 1+\epsilon$ for $|n| \leq N$. Passing to the limit as $N \rightarrow \infty$ and $\epsilon \rightarrow 0$ we get the desired eigenfunction 8

Remark 3.4. The result can be generalized to some classes of continuous ergodic operators. For dynamical systems, corresponding results appear in the work of Mañé. Namely, if a cocycle is not uniformly hyperbolic then there exists a vector that is never expanded, either in the future or in the past.

\subsection{Main dynamical estimates: quantitative Aubry duality}

We fix $\alpha, v, \lambda$ as in Theorem 3.2 For every $E \in \Sigma_{\lambda v, \alpha}$, let $\theta=\theta(E)$ be given by Theorem $3.3^{9}$ Let $\left\{n_{j}\right\}$ be the set of resonances of $\theta(E)$. Let $A=S_{\lambda v, E}$. In what follows, $C$ is a large constant and $c$ is a small constant, which are allowed to depend on $v, \lambda, \alpha$, but not on $E$ or $\theta$.

Theorem 3.4. Fix some $n=\left|n_{j}\right|+1<\infty$ and let $N=\left|n_{j+1}\right|$. Then there exists $\Phi: \mathbb{R} / \mathbb{Z} \rightarrow \operatorname{SL}(2, \mathbb{C})$ analytic with $\|\Phi\|_{c n^{-C}} \leq C n^{C}$ such that

$$
\Phi(x+\alpha) A(x) \Phi(x)^{-1}=\left(\begin{array}{cc}
e^{2 \pi i \theta} & 0 \\
0 & e^{-2 \pi i \theta}
\end{array}\right)+\left(\begin{array}{cc}
q_{1}(x) & q(x) \\
q_{3}(x) & q_{4}(x)
\end{array}\right)
$$

with

$$
\left\|q_{1}\right\|_{c n^{-C}},\left\|q_{3}\right\|_{c n^{-C}},\left\|q_{4}\right\|_{c n^{-C}} \leq C e^{-c N}
$$

and

$$
\|q\|_{c n^{-C}} \leq C e^{-c n(\ln (1+n))^{-C}} .
$$

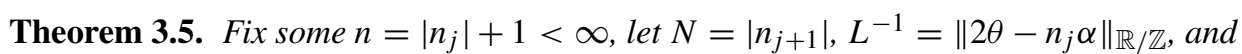
assume that $0<L^{-1}<c .10$ Then there exists $W: \mathbb{R} / \mathbb{Z} \rightarrow \operatorname{SL}(2, \mathbb{R})$ analytic such that $|\operatorname{deg} W| \leq C n,\|W\|_{c} \leq C L^{C}$ and $\left\|W(x+\alpha) A(x) W(x)^{-1}-R_{\mp \theta}\right\|_{c} \leq C e^{-c N}$.

Remark 3.5. For $N=\infty$, Theorem 3.5 gives a quantitative version of the first statement of Theorem 2.5 .

We will prove Theorem 3.4 in $\$ 7$ and Theorem 3.5 in $\$ 8$ All spectral results are consequences of those theorems combined with Theorem 3.2

\footnotetext{
8 An alternative proof is the following. Let $p / q$ be close to $\alpha$, and let $\theta^{\prime} \in \mathbb{R}$. The spectrum of $\hat{H}^{\prime}=\hat{H}_{v, p / q, \theta^{\prime}}$ is close to the spectrum of $\hat{H}$ in the Hausdorff topology. Let $E^{\prime}$ be close to $E$ and in the spectrum of $\hat{H}^{\prime}$. Then there is a non-zero periodic solution to $\hat{H}^{\prime} \hat{u}^{\prime}=E^{\prime} \hat{u}^{\prime}$. Changing $\theta^{\prime}$ to $\theta^{\prime}+k p / q$, we may assume that $\hat{u}_{0}^{\prime}=1$ and $\left|\hat{u}_{n}^{\prime}\right| \leq 1$. Taking the limit $p / q \rightarrow \alpha, E^{\prime} \rightarrow E$, a limit $\theta$ of $\theta^{\prime}$, and a pointwise limit $\hat{u}$ of $\hat{u}^{\prime}$, we get the statement.

9 Notice that $\theta(E)$ is not necessarily uniquely defined. There could be uncountably many resonant $\theta$, but at most finitely many non-resonant $\theta$, corresponding to the same $E$. This does not concern our arguments, we just fix some $\theta$.

10 It is likely that the result holds assuming only $L^{-1}>0$.
} 


\subsection{Outline of the rest of the paper}

Almost reducibility (Theorem 1.4) and the direct proof of Theorem 1.1 are immediate consequences of Theorem 3.5 Estimates related to modulus of continuity, including Theorem 1.6, are obtained from Theorem 3.4 All are more or less immediate. We will discuss those consequences in $\$ 4$

The technical core of the paper is formed by the proofs of Theorems $3.2,3.4$ and 3.5 The dynamical estimates build on preliminary estimates and ideas developed in $\$ 6$, but are otherwise independent. Localization $(\$ 5)$ and quantitative duality $(\$ \$ 6,7,7$, and 8$)$ are independent. Section 5 uses the machinery developed in [BJ1] and some shortcuts from [AJ1]. The techniques and ideas developed in $\$ \$ 6,7$, and 8 are new and do not use any ideas/methods from the existing literature.

\section{Easy spectral consequences of the main dynamical estimates}

\subsection{Almost reducibility}

We will show the following precise version of Theorem 1.4

Theorem 4.1. Assume $\alpha \in \mathrm{DC}$ and $0<|\lambda|<\lambda_{0}(v)$. There exists $c>0$ (depending on $\lambda, v, \alpha)$ with the following property. Let $A=S_{\lambda v, E}$.

(1) If $\rho(\alpha, A)$ is c-resonant then there exists a sequence $B^{(n)}: \mathbb{R} / \mathbb{Z} \rightarrow \operatorname{SL}(2, \mathbb{R})$ such that $B^{(n)}(x+\alpha) A(x) B^{(n)}(x)^{-1}$ converges to a constant rotation uniformly in the set $\{|\Im x|<c\}$.

(2) If $\rho(\alpha, A)$ is not c-resonant and $2 \rho(\alpha, A) \notin \alpha \mathbb{Z}+\mathbb{Z}$ then there exists $B: \mathbb{R} / \mathbb{Z} \rightarrow$ $\mathrm{SL}(2, \mathbb{R})$, analytically extending to $\{|\Im z|<c\}$, such that $B(x+\alpha) A(x) B(x)^{-1}$ is a constant rotation.

(3) If $2 \rho(\alpha, A) \in \alpha \mathbb{Z}+\mathbb{Z}$ then there exists $B: \mathbb{R} / \mathbb{Z} \rightarrow \operatorname{PSL}(2, \mathbb{R})$ analytic such that $B(x+\alpha) A(x) B(x)^{-1}$ is a constant.

Proof. If $E \notin \Sigma$, by Theorem 2.2, $2 \rho(\alpha, A) \in \alpha \mathbb{Z}+\mathbb{Z}$, and by Theorem 2.1. $(\alpha, A)$ is reducible.

Let $E \in \Sigma$. If $\theta$ is not $\epsilon_{0}$-resonant, by Theorem 3.2 there is an exponentially decaying eigenfunction. Theorem 2.5 thus applies, and the result holds in all cases (using Remark 2.2 .

Assume that $\theta$ is $\epsilon_{0}$-resonant (and thus $2 \theta \notin \alpha \mathbb{Z}+\mathbb{Z}$ ). Applying Theorem 3.5 . we get for every $j$ a matrix function $B^{(j)}: \mathbb{R} / \mathbb{Z} \rightarrow \operatorname{SL}(2, \mathbb{R})$ such that $\Phi(x)=$ $B^{(j)}(x+\alpha) A(x) B^{(j)}(x)^{-1}$ satisfies $\left\|\Phi(x)-R_{\mp \theta}\right\|_{c} \leq C e^{-c N}$ and $\left|\operatorname{deg} B^{(j)}\right| \leq$ $C\left(\left|n_{j}\right|+1\right)$ (where $\left.N=\left|n_{j+1}\right|\right)$. To conclude, let us show that $\rho$ is $c$-resonant.

By 2.9 , $\left|\rho(\alpha, A) \pm \theta+\left(\operatorname{deg} B^{(j)}\right) \alpha\right| \leq C e^{-c N}$. Using 2.8 and Lemma 3.1 we get, for large $j$,

$\left\|2 \rho(\alpha, A) \pm\left(n_{j} \pm 2 \operatorname{deg} B^{(j)}\right) \alpha\right\|_{\mathbb{R} / \mathbb{Z}} \geq\left\|\mp 2 \theta \pm n_{j} \alpha\right\|_{\mathbb{R} / \mathbb{Z}}-C e^{-c N} \geq c N^{-C}-C e^{-c N}>0$, 


$$
\begin{aligned}
\left\|2 \rho(\alpha, A) \pm\left(n_{j} \pm 2 \operatorname{deg} B^{(j)}\right) \alpha\right\|_{\mathbb{R} / \mathbb{Z}} \leq\left\|\mp 2 \theta \pm n_{j} \alpha\right\|_{\mathbb{R} / \mathbb{Z}}+C e^{-c N} \\
\leq e^{-\epsilon_{0}\left|n_{j}\right|}+C e^{-c N} \leq e^{-c\left|n_{j}\right|} e^{-c\left(\left|n_{j} \pm 2 \operatorname{deg} B^{(j)}\right|\right)} .
\end{aligned}
$$

For $j$ large, (4.2) and the Diophantine condition imply that $\rho(\alpha, A)$ has a $c$-resonance at $\mp\left(n_{j} \pm 2 \mathrm{deg} B^{(j)}\right)$. If the set of $c$-resonances for $\rho(\alpha, A)$ were not infinite, then we would have $\left\|2 \rho(\alpha, A) \pm\left(n_{j} \pm 2 \operatorname{deg} B^{(j)}\right) \alpha\right\|_{\mathbb{R} / \mathbb{Z}}=0$ for large $j$. But this contradicts 4.1 .

Remark 4.1. This result is new even in the perturbative regime. Though Eliasson's scheme provides a sequence of approximate conjugacies to normal forms, for badly behaved $\rho$ there is only control in a shrinking sequence of bands, as is typical for KAM analysis. In fact, it does not imply our strong definition of almost reducibility of cocycles except when it actually implies reducibility.

Remark 4.2. The analytic conjugacy given in the third statement for $E \in \Sigma$ can be shown to extend also to a definite strip. For $E \notin \Sigma$, we do not get any estimates on the analytic extension.

We also state separately the following statement, already obtained as a part of the proof of Theorem 4.1. By Theorem 2.5, if $\theta(E)$ (as specified in Theorem 3.3, see also footnote 9 in Section 3.3 is not $\epsilon_{0}$-resonant, then $\rho(E)= \pm \theta+k \alpha / 2$ for some $k$. There are reasons to believe that this does not hold for all $E$ or $\theta$ Instead, for resonant $\theta$ we have the following statement.

Theorem 4.2. Fix $E \in \Sigma$ and some $\theta(E)$. Under the conditions of Theorem 4.1 and with the same $c$, if $\theta$ is $\epsilon_{0}$-resonant, then $\rho$ is c-resonant.

\subsection{Open gaps for the almost Mathieu operator (direct proof)}

By Theorem 2.2 in the closure of a component of $\mathbb{R} \backslash \Sigma$ we must have $N(E)=1-2 \rho \in$ $\alpha \mathbb{Z}+\mathbb{Z}$. The dry Ten Martini Problem is the conjecture that the converse holds in the case of the almost Mathieu operator. Since the integrated density of states is the same for $H$ and $\hat{H}$, it does not matter whether we prove the statement for $\lambda$ or for $\lambda^{-1}$. Theorem 1.1 is thus a consequence of the following.

Theorem 4.3. Let $v(x)=2 \cos 2 \pi x$, and let $0<|\lambda|<1$ and $\alpha$ Diophantine. If $E \in \Sigma$ is such that $N(E) \in \alpha \mathbb{Z}+\mathbb{Z}$ then $E$ belongs to the boundary of a component of $\mathbb{R} \backslash \Sigma$.

Proof. By [P1], it is enough to show that $\left(\alpha, S_{\lambda v, E}\right)$ is reducible. By Theorem 4.1, if this were not the case then $\rho\left(\alpha, S_{\lambda v, E}\right)$ would be $c$-resonant. By the Diophantine condition and Remark 3.2, $2 \rho \notin \alpha \mathbb{Z}+\mathbb{Z}$. Since $N=1-2 \rho$, this is a contradiction.

\footnotetext{
11 Otherwise the set of $E$ 's corresponding to a given $\theta$ would be countable even when $\hat{H}_{\theta}$ has singular continuous spectrum. If we relaxed slightly the definition of $\theta(E)$ to require $\left|\hat{u}_{0}\right|>1-\epsilon$ instead of $\left|\hat{u}_{0}\right|=1$ we could have argued that this is a contradiction since it can be shown in certain cases that all generalized eigenfunctions are bounded, and therefore for all energies $E$ in the support of singular-continuous measure for a given $\theta$ one can find $\theta(E)$ of the form $\theta+k \alpha$ for some $k$. Such relaxed definition of $\theta(E)$ could also be used in our proofs, with only small changes.
} 


\subsection{Precise bounds on growth, complex perturbations}

Conventions below are as in Section 3.3

Theorem 4.4. Let $n=\left|n_{j}\right|+1<\infty$ and let $N=\left|n_{j+1}\right|$. There exists $W=W_{(\epsilon)}$ : $\mathbb{R} / \mathbb{Z} \rightarrow \operatorname{SL}(2, \mathbb{C})$ analytic such that, letting $Z(x)=Z_{(\epsilon)}(x)=W_{(\epsilon)}(x+\alpha) A(x) W_{(\epsilon)}^{-1}(x)$, we have

$$
\begin{aligned}
& \|W\|_{c n^{-C}} \leq C \epsilon^{-1 / 4} \text { and }\|Z\|_{c n^{-C}} \leq 1+C e^{-c n(\ln (n+1))^{-C}} \epsilon^{1 / 2} \\
& \quad \text { for } C e^{-c N} \leq \epsilon \leq c n^{-C} .
\end{aligned}
$$

Proof. Let $\Phi$ be given by Theorem 3.4. Let $D=\left(\begin{array}{cc}d & 0 \\ 0 & d^{-1}\end{array}\right)$ where $d=\|\Phi\|_{c n^{-C}} \epsilon^{1 / 4}$. Let $W(x)=D \Phi(x)$. If $\epsilon \leq c n^{-C}$ we have $\|W\|_{c n^{-C}} \leq C \epsilon^{-1 / 4}$. Since

$$
D\left(\begin{array}{ll}
a_{1} & a_{2} \\
a_{3} & a_{4}
\end{array}\right) D^{-1}=\left(\begin{array}{cc}
a_{1} & d^{2} a_{2} \\
d^{-2} a_{3} & a_{4}
\end{array}\right)
$$

we get

$$
Z(x)=W(x+\alpha) A(x) W(x)^{-1}=\left(\begin{array}{cc}
e^{2 \pi i \theta} & 0 \\
0 & e^{-2 \pi i \theta}
\end{array}\right)+\left(\begin{array}{cc}
z_{1}(x) & z_{2}(x) \\
z_{3}(x) & z_{4}(x)
\end{array}\right)
$$

with $\left\|z_{1}\right\|_{c n^{-C}},\left\|z_{3}\right\|_{c n^{-C}},\left\|z_{4}\right\|_{c n^{-C}} \leq C \epsilon^{-1 / 2} e^{-c N}$ and $\left\|z_{2}\right\|_{c n^{-C}} \leq C \epsilon^{1 / 2} e^{-c n(\ln (n+1))^{-C}}$. If $\epsilon \geq C e^{-c N}$ then $\|Z\|_{c n^{-C}} \leq 1+C e^{-c n(\ln (n+1))^{-C}} \epsilon^{1 / 2}$.

The following gives a direct proof of a perturbative estimate of [E] (see Theorem A.1] in the Appendix).

Corollary 4.5. For every $s \geq 0$ we have $\left\|A_{s}\right\|_{0} \leq C(1+s)$. Moreover, if $\theta$ is non-rational then $\left\|A_{s}\right\|_{0}=o(1+s)$.

Proof. By Lemma 3.1, for every $s \geq C, \epsilon=1 / s^{2}$ is in the range specified in 4.3 for some choice of $n=\left|n_{j}\right|+1$. Then

$\ln \left\|A_{S}\right\|_{0} \leq 2 \ln \|W\|_{0}+s \ln \|Z\|_{0} \leq C-\frac{1}{2} \ln \epsilon+s C e^{-c n(\ln (n+1))^{-C}} \epsilon^{1 / 2} \leq C+\ln (1+s)$.

This gives $\left\|A_{s}\right\|_{0} \leq C(1+s)$.

If $\theta$ is non-rational and non-resonant then by Theorems 3.2 and $2.5 .\left\|A_{s}\right\|=O(1)$. If $\theta$ is resonant then we have $n \rightarrow \infty$ as $s \rightarrow \infty$, and we get the estimate

$$
\sup _{0 \leq j \leq c e^{c n(\ln n)-C} s} \ln \left\|A_{j}\right\| \leq C+\ln (1+s),
$$

which implies the second statement.

Corollary 4.6. If $B: \mathbb{R} / \mathbb{Z} \rightarrow \operatorname{SL}(2, \mathbb{C})$ is continuous then $L(\alpha, B) \leq C\|B-A\|_{0}^{1 / 2}$. 
Proof. It is enough to consider the case when $\epsilon=\|B-A\|_{0}$ is sufficiently small. Then $\epsilon$ is in the range specified by $(4.3)$ for some $n=\left|n_{j}\right|+1$. Let $\tilde{B}(x)=W(x+\alpha) B(x) W(x)^{-1}$. Then $\|\tilde{B}\|_{0} \leq\|Z\|_{0}+\|W\|_{0}^{2}\|B-A\|_{0} \leq 1+C \epsilon^{1 / 2}$. Hence $L(\alpha, B)=L(\alpha, \tilde{B}) \leq$ $\ln \|\tilde{B}\|_{0} \leq C \epsilon^{1 / 2}$.

As before, the estimate is improved when $\theta$ is not rational.

The integrated density of states: direct proof of Theorem 1.6 Theorem 1.6 follows easily from Corollary 4.6. Indeed, $L(E)=\int \ln \left|E-E^{\prime}\right| d N\left(E^{\prime}\right)$ by the Thouless formula. Thus

$$
\begin{aligned}
L(E+i \epsilon) & \geq L(E+i \epsilon)-L(E)=\frac{1}{2} \int \ln \left(1+\frac{\epsilon^{2}}{\left(E-E^{\prime}\right)^{2}}\right) d N\left(E^{\prime}\right) \\
& \geq c(N(E+\epsilon)-N(E-\epsilon))
\end{aligned}
$$

for every $\epsilon>0$. By Corollary $4.6, L(E+i \epsilon) \leq C \epsilon^{1 / 2}$ for $E \in \Sigma$. Thus $N(E+\epsilon)-$ $N(E-\epsilon) \geq C c^{-1} \epsilon^{1 / 2}$ for every $0<\epsilon<1, E \in \Sigma$. Since $N$ is locally constant in the complement of $\Sigma$, this means precisely that $N$ is $1 / 2$-Hölder.

\section{Almost localization: proof of Theorem 3.2}

Although it will not be needed for the rest of the paper, we will consider a weaker Diophantine condition on $\alpha$. For $\nu, \xi>0$, let $\operatorname{EDC}(v, \xi)$ be the set of $\alpha$ such that

$$
|q \alpha-p| \geq v e^{-\xi q}, \quad p \in \mathbb{Z}, q \in \mathbb{Z} \backslash\{0\} .
$$

Clearly for all $\kappa, \tau, \xi>0$ there exists $v>0$ such that $\operatorname{DC}(\kappa, \tau) \subset \operatorname{EDC}(\nu, \xi)$. If $\alpha \in$ $\operatorname{EDC}(v, \xi)$ then, by [2.15), $\ln q_{n+1} \leq \xi q_{n}-\ln v$.

We will prove the following precise version of Theorem 3.2

Theorem 5.1. There exists $\lambda_{0}(v)>0$ such that if $0<\lambda<\lambda_{0}$ and $C_{0}>1$, then there exist $\xi=\xi\left(\lambda, v, C_{0}\right)>0, \epsilon_{0}=\epsilon_{0}(v, \lambda)>0, \epsilon_{1}=\epsilon_{1}\left(v, \lambda, C_{0}\right)>0$ such that if $\alpha \in$ $\operatorname{EDC}(\nu, \xi)$, then the family $\hat{H}_{\lambda v, \alpha, \theta}$ is almost localized with parameters $C_{0}, \epsilon_{0}, \epsilon_{1}, C_{1}$, where $C_{1}=C_{1}\left(v, \lambda, C_{0}, v\right)>0$. For $v(x)= \pm 2 \cos 2 \pi x, \lambda_{0}=1$.

Proof of Theorem 5.1. To simplify the notation, fix some $v: \mathbb{R} / \mathbb{Z} \rightarrow \mathbb{R}$ analytic, and set $\check{H}=\breve{H}_{\alpha, \theta}=(1 / \lambda) \hat{H}_{\lambda v, \alpha, \theta}$. Throughout this section, $C$ denotes an absolute large constant, while $C_{\sigma}$, for instance, denotes a large constant that only depends on $\sigma$. We warn that this is not the same convention used in the rest of this paper.

Fix an interval $I \subset \mathbb{Z}$. Let $\Gamma$ be the coupling operator between $I$ and $\mathbb{Z} \backslash I$ :

$$
\Gamma(i, j)= \begin{cases}\hat{v}_{i-j}, & \chi_{I}(i)+\chi_{I}(j)=1, \\ 0, & \text { otherwise. }\end{cases}
$$

Then we can write $\hat{u}=-(\check{H}-E-\Gamma)^{-1} \Gamma \hat{u}$, from which for any $x \in I$ we obtain

$$
\hat{u}(x)=-\sum_{y \in I, k \notin I} G_{I}(x, y) \hat{v}_{y-k} \hat{u}(k),
$$


where $G_{I}$ is the Green's function of $\check{H}$ restricted to the interval $I, G_{I}=G_{I}(E)=$ $\left(R_{I}(\breve{H}-E) R_{I}^{*}\right)^{-1}\left(\right.$ where $R_{I}: l^{2}(\mathbb{Z}) \rightarrow l^{2}(I)$ and $R_{I}^{*}: l^{2}(I) \rightarrow l^{2}(\mathbb{Z})$ denote the restriction and the inclusion operators). Set

$$
a_{k}=\sum_{j:|j| \geq|k|, j k \geq 0}\left|j \hat{v}_{j}\right| .
$$

Fix $E, m \in \mathbb{R}$. A point $y \in \mathbb{Z}$ will be called $(m, k)$-regular if there exists an interval $I=\left[x_{1}+1, x_{2}-1\right]$ with $x_{2}=x_{1}+k+1$ such that $x_{1}<y<x_{2}$ and

$$
\sum_{x \in I, i=1,2}\left|G_{I}(y, x) a_{x-x_{i}}\right|<e^{-m k} .
$$

Otherwise, $y$ will be called $(m, k)$-singular.

The strategy is to show that the existence of a generalized eigenfunction as in Definition 3.1 implies that $k$ is $(m, \ell(k))$-regular for an appropriate $m>0$ and for $\ell(k)$ comparable with $k$, with $k$ in the desired "between the resonances" region.

Define $I \subset \mathbb{Z}$ by $I=[0, N-1], N \in \mathbb{N}$. We will omit the $\alpha, E$ dependence of various quantities in what follows, and all constants will be uniform for all $E$ in the spectrum. We will also often assume $v \not \equiv 0$, as otherwise our statements become trivial. Let $P_{N}(\theta)=\operatorname{det} R_{I}\left(\check{H}_{\alpha, \theta}-E\right) R_{I}^{*}$. Then $P_{N}(\theta)$ is an even function of $\theta+\frac{N-1}{2} \alpha$ and can be written as a polynomial of degree $N$ in $\cos 2 \pi\left(\theta+\frac{N-1}{2} \alpha\right)$ :

$$
P_{N}(\theta)=\sum_{j=0}^{N} c_{j} \cos ^{j} 2 \pi\left(\theta+\frac{N-1}{2} \alpha\right) \stackrel{\text { def }}{=} Q_{N}\left(\cos 2 \pi\left(\theta+\frac{N-1}{2} \alpha\right)\right) .
$$

Lemma 5.2. $\int_{0}^{1} N^{-1} \ln \left|P_{N}(\theta)\right| d \theta \geq-\ln \lambda$.

Proof. This is proved by a standard Herman's subharmonicity argument $[\mathrm{H}]$.

Let $A_{k, r}=\left\{\theta \in \mathbb{R}:\left|Q_{k}(\cos 2 \pi \theta)\right| \leq e^{(k+1) r}\right\}$. The next lemma shows that every singular point "produces" a long piece of the trajectory of the rotation consisting of points belonging to an appropriate $A_{k, r}$. It is fairly immediate in the almost Mathieu case, and we will adapt the argument of [BJ1] for the general case.

Lemma 5.3. There exists $\lambda_{0}=\lambda_{0}(v)>0$ such that for $0<\lambda<\lambda_{0}, 1 / 40 \leq \delta<1 / 2$, 12 $\epsilon=\epsilon(\lambda, v)>0, c=c(\lambda, v)>0, K \subset \mathbb{R} \backslash \mathbb{Q}$ compact, $\alpha \in K$, if $y \in \mathbb{Z}$ is $(c, N)$ singular, $N>N(\lambda, v, K)$ and $x \in \mathbb{Z}$ is such that $y-(1-\delta) N \leq x \leq y-\delta N$, then $\theta+\left(x+\frac{N-1}{2}\right) \alpha$ belongs to $A_{N,-\ln \lambda-\epsilon}$. For $v(x)= \pm 2 \cos 2 \pi x, \lambda_{0}=1$.

Assume, without loss of generality, that $C_{0}\left|n_{j_{k}}\right|<k<\left(1 / C_{0}\right)\left|n_{j_{k}+1}\right|$, and $k$ is large (depending only on $C_{0}$ ). We will define scales $n \geq 0$ and $s \geq 1$ associated with $k$ so that

$$
2 s q_{n} \leq \zeta k<\min \left\{2(s+1) q_{n}, 2 q_{n+1}\right\},
$$

where $\zeta=1 / 32$ if $2\left|n_{j_{k}}\right|<k<\left|n_{j_{k}+1}\right| / 2$ and $\zeta=\left(C_{0}-1\right) /\left(16 C_{0}\right)$ otherwise. Note that $s, n$ depend on $\epsilon_{0}$.

12 As will be clear from the proof, the result still holds for any $0<\delta<1 / 2$, but the constants, including $\lambda_{0}$, become dependent on $\delta$. 
Lemma 5.4. Assume there exists $\hat{u}$ as in Definition 3.1 For $\lambda$, $c$ as in Lemma 5.3 there exist $\xi=\xi\left(v, \lambda, C_{0}\right)>0, \epsilon_{0}=\epsilon_{0}(v, \lambda)>0$ such that if $\alpha \in \operatorname{EDC}(v, \xi), k>$ $k\left(v, \lambda, c, C_{0}, \nu, \xi\right)$ and $s, n$ are as above then $k$ is $\left(c, 6 s q_{n}-1\right)$-regular.

Since $s q_{n}>C_{C_{0}}^{-1} k$ the theorem now follows immediately from the definition of regularity and 5.2. It therefore suffices to prove Lemmas 5.3 and 5.4

Proof of Lemma 5.3. Without loss, set $x=0$. Set $x_{1}=-1, x_{2}=N$. Assume $\theta+$ $\frac{N-1}{2} \alpha \notin A_{N,-\ln \lambda-\epsilon}$, that is, $P_{N}(\theta)>\lambda^{N} e^{-\epsilon N}$. We need to show that for $y \in\left[x_{1}, x_{2}\right]$ with $\operatorname{dist}\left(y, \partial\left[x_{1}, x_{2}\right]\right) \geq \delta N$ we have

$$
(*)=\sum_{z \in I, i=1,2}\left|G_{I}(y, z)\right|\left|a_{z-x_{i}}\right|<e^{-c N} .
$$

By Cramer's rule $G_{I}(y, z)=\mu_{y, z} / P_{N}(\theta)$ where $\mu_{y, z}$ is the corresponding minor. The following lemma reduces the study of Green's function to the study of determinants of restrictions of $\check{H}-E$.

Lemma 5.5 (Lemma 10 of [B]1]).

$$
\mu_{y, z}=\sum_{\gamma} \alpha_{\gamma} \operatorname{det} R_{I \backslash \gamma}(\check{H}-E) R_{I \backslash \gamma}^{*} \prod_{i=1}^{|\gamma|}\left|\hat{v}_{\gamma_{i+1}-\gamma_{i}}\right|
$$

where the sum is taken over all ordered subsets $\gamma=\left(\gamma_{1}, \ldots, \gamma_{n}\right)$ of $I$ with $\gamma_{1}=y$ and $\gamma_{n}=z,|\gamma|=n-1$, and $\alpha_{\gamma} \in\{-1,1\}$.

The following is a simplified, yet more general, version of Lemma 11 of [BJ1]. It gives an upper bound on $\mu_{y, z}$.

Lemma 5.6. For any $\Lambda \subset I$, for sufficiently large $N>N(v, \lambda, K)$,

$$
\left|\operatorname{det} R_{I \backslash \Lambda}(\check{H}-E) R_{I \backslash \Lambda}^{*}\right| \leq \lambda^{-N} e^{C\|v\|_{0}^{1 / 2} \lambda^{1 / 2} N}\left(\|v\|_{0}+C^{-1} \lambda^{-1} \frac{\# \Lambda^{2}}{N^{2}}\right)^{-\# \Lambda} .
$$

Remark 5.1. Unlike the corresponding upper bound in [BJ1], this lemma does not require $\alpha \in \mathrm{DC}$.

Proof. By Hadamard's bound, we have

$$
\left|\operatorname{det} R_{I \backslash \Lambda}(\check{H}-E) R_{I \backslash \Lambda}^{*}\right| \leq \prod_{j \in I \backslash \Lambda}\left(\left(2 \lambda^{-1} \cos 2 \pi(\theta+j \alpha)-E\right)^{2}+\|v\|_{L^{2}}^{2}\right)^{1 / 2} .
$$

Thus

$$
\begin{aligned}
\ln \left|\operatorname{det} R_{I \backslash \Lambda}(\check{H}-E) R_{I \backslash \Lambda}^{*}\right| & \leq \sum_{j \in I \backslash \Lambda} \frac{1}{2} \ln \left(\left(2 \lambda^{-1} \cos 2 \pi(\theta+j \alpha)-E\right)^{2}+\|v\|_{0}^{2}\right) \\
& =\sum_{j \in I \backslash \Lambda} u(x+j \alpha) .
\end{aligned}
$$


Set $A_{t}=\{x:|2 \cos 2 \pi x-\lambda E|<t\}$ for $0 \leq t \leq 2+|\lambda E|$, and let $t_{0}$ be such that the Lebesgue measure of $A_{t_{0}}$ is equal to $\# \Lambda / N$. Then by unique ergodicity of irrational rotations and continuity of $u$, we have

$$
\begin{aligned}
\sum_{j \in I \backslash \Lambda} u(x+j \alpha) & \leq \sum_{j \in I} u(x+j \alpha)-\sum_{\substack{j \in I \\
x+j \alpha \in A_{t_{0}}}} u(x+j \alpha)+N o(1) \\
& \leq N\left(\int u(x)\left(1-\chi_{A_{t_{0}}}\right) d x+o(1)\right) .
\end{aligned}
$$

Notice that a direct computation gives

$$
\int u(x) d x=\ln \lambda^{-1}+|\ln | z+\sqrt{z^{2}-1}||
$$

where $z=\left(E+i\|v\|_{0}\right) /\left(2 \lambda^{-1}\right)$ (see [BJ1] for details). From this and $|E| \leq 2 \lambda^{-1}+\|v\|_{0}$ in the spectrum, we get the estimate $\int u(x) d x \leq \ln \lambda^{-1}+C \lambda^{1 / 2}\|v\|_{0}^{1 / 2}$. To complete the proof, it suffices to show that, letting $s=\# \Lambda / N$,

$$
\int u(x) \chi_{A_{t_{0}}}(x) d x \geq s \ln \left(\|v\|_{0}+C^{-1} \lambda^{-1} s^{2}\right)-C\|v\|_{0}^{1 / 2} \lambda^{1 / 2} .
$$

Let us split $A_{t}$ into four segments $I_{j}$ of length $s_{j} \leq 1$ such that $x \mapsto(2 \cos 2 \pi x-\lambda E)^{2}$ is monotonic in $I_{j}$. Then, since $1-\cos 2 \pi x \geq 16 x^{2}$ for $x \leq 1 / 4$,

$$
\begin{aligned}
\int_{I_{j}} u(x) d x & \geq \int_{0}^{s_{j}} \frac{1}{2} \ln \left(\|v\|_{0}^{2}+4 \lambda^{-2}(1-\cos 2 \pi x)^{2}\right) d x \\
& \geq \int_{0}^{s_{j}} \frac{1}{2} \ln \left(\|v\|_{0}^{2}+256 \lambda^{-2} x^{4}\right) d x \\
& \geq \frac{1}{2} s_{j} \ln \left(\|v\|_{0}^{2}+256 \lambda^{-2} s_{j}^{4}\right)-2 s_{j} .
\end{aligned}
$$

Since $x \mapsto x \ln \left(\|v\|_{0}^{2}+256 \lambda^{-2} x^{4}\right)$ is concave on $\mathbb{R}^{+}$, we get, summing over $j$,

$$
\int_{A_{t_{0}}} u(x) d x \geq \frac{1}{2} s \ln \left(\|v\|_{0}^{2}+\lambda^{-2} s^{4}\right)-2 s .
$$

Considering separately the cases $\lambda^{-1} s^{2}>c\|v\|_{0}$ and $\lambda^{-1} s^{2}<c\|v\|_{0}$ we conclude that this implies (5.11).

Our assumption $\left|P_{N}(\theta)\right|>\lambda^{-N} e^{-\epsilon N}$ implies, with the notation of 5.4 and using 5.5,

$$
\begin{aligned}
(*) & \leq\left(\lambda e^{\epsilon}\right)^{N} \sum_{n=1}^{N-1} \sum_{\substack{i=1,2 \\
\gamma:|\gamma|=n}}\left|\operatorname{det} R_{I \backslash \gamma}(\check{H}-E) R_{I \backslash \gamma}^{*}\right|\left|a_{x_{i}-\gamma_{|\gamma|+1}}\right| \prod_{i=1}^{n}\left|\hat{v}_{\gamma_{i+1}-\gamma_{i}}\right| \\
& \leq e^{\left(\epsilon+C\|v\|_{0}^{1 / 2} \lambda^{1 / 2}\right) N} \sum_{n=1}^{N-1} \sum_{\substack{i=1,2 \\
\gamma:|\gamma|=n}} C_{\sigma} C_{v, \sigma}^{n+1}\left(\|v\|_{0}+C^{-1} \lambda^{-1} \frac{(n+1)^{2}}{N^{2}}\right)^{-(n+1)} e^{-\sigma b(\gamma, i)},
\end{aligned}
$$


where $0<\sigma<\sigma(v) \leq 1$ is such that

$$
\left|\hat{v}_{k}\right| \leq C_{v, \sigma} e^{-|k| \sigma},
$$

and $b(\gamma, i)=|\gamma| \gamma\left|+1-x_{i}\right|+\sum_{i=1}^{|\gamma|}\left|\gamma_{i+1}-\gamma_{i}\right|$. Let $G_{b, n}=\{\gamma:|\gamma|=n$ and $b(\gamma, i)=b\}$. Then

$$
\begin{aligned}
(*) & \leq e^{\left(\epsilon+C\|v\|_{0}^{1 / 2} \lambda^{1 / 2}\right) N} \sum_{n=1}^{N-1} \sum_{b} C_{\sigma} C_{v, \sigma}^{n+1}\left(\|v\|_{0}+C^{-1} \lambda^{-1} \frac{(n+1)^{2}}{N^{2}}\right)^{-(n+1)} e^{-\sigma b} \# G_{b, n} \\
& \leq e^{\left(\epsilon+C\|v\|_{0}^{1 / 2} \lambda^{1 / 2}\right) N} \sum_{n=1}^{N-1} C_{\sigma}\left(2 C_{v, \sigma}\right)^{n+1}\left(C^{-1} \lambda^{-1} \frac{(n+1)^{2}}{N^{2}}\right)^{-(n+1)} \sum_{b, G_{b, n} \neq \emptyset} e^{-\sigma b}\left(\begin{array}{l}
b \\
n
\end{array}\right) .
\end{aligned}
$$

If $G_{b, n} \neq \emptyset$ then $\delta N \leq \max \{\operatorname{dist}(y, \partial I), n+1\} \leq b \leq(n+1) N \leq N^{2}$. By Stirling's formula, setting $b=r N, n+1=s b$, we have $\left(\begin{array}{l}b \\ n\end{array}\right) \leq C r N e^{\phi(s) r N}$ where $\phi(s)=$ $-s \ln s-(1-s) \ln (1-s)$. Thus we can estimate

$$
(*) \leq e^{\left(\epsilon+C\|v\|_{0}^{1 / 2} \lambda^{1 / 2}\right) N} C C_{\sigma} N^{5} \sup _{\substack{\delta \leq r \leq n+1 \\ 0<s \leq 1}}\left(\frac{C^{-1} \lambda^{-1}}{2 C_{v, \sigma}} r^{2} s^{2}\right)^{-r s N} e^{-\sigma r N} e^{\phi(s) r N} .
$$

The desired exponential decay of $(*)$ follows if

$$
(* *)=\sup _{0<s \leq 1} \epsilon+C\|v\|_{0}^{1 / 2} \lambda^{1 / 2}+\left(\ln 2 C+\ln C_{v, \sigma}+\ln \lambda-2 \ln \delta s-\frac{\sigma}{s}+\frac{\phi(s)}{s}\right) \delta s<0 .
$$

This condition is satisfied (for appropriate $\epsilon$ ) if

$$
c_{0}=\delta^{-2} \lambda C_{v, \sigma} \sigma^{-3}
$$

is small. Indeed, since $\|v\|_{0} \leq C C_{v, \sigma} / \sigma$ and $\phi(s) / s \leq 1-\ln s$, we have

$$
(* *) \leq \epsilon+\left(C \delta c_{0}^{1 / 2}-\frac{\delta}{2}\right) \sigma+\left(\ln C+\ln c_{0}+3 \ln \frac{\sigma}{s}-\frac{\sigma}{2 s}\right) \delta s .
$$

In case $v(x)=2 \cos 2 \pi x$ the result in the form we need, for any $0<\lambda<1$, is stated in Lemma 9.2 in [AJ1] (uniformity on $E$ was not claimed but is automatic from the argument). The simplification in this case is mainly due to the fact that, as can be seen by a direct computation (but also follows immediately from Lemma 5.5), $\left|\mu_{y, z}\right|=$ $\left|P_{y-1}(\theta) P_{N-z}(\theta+z \alpha)\right|$ and we have a uniform upper bound of the form $\ln \left|P_{k}(\theta)\right|<$ $C_{\epsilon, K}+k\left(\ln \lambda^{-1}+\epsilon\right)$. It is obtained by identifying $P_{k}(\theta)$ with the upper-left coefficient of the $k$-th iterate of the almost Mathieu cocycle $\left(\alpha, S_{\lambda-1} v, E\right)$, and applying the formula $L\left(\alpha, S_{\lambda^{-1}, E}\right)=-\ln \lambda$ for $0<\lambda<1$ and all $E$ in the spectrum [BJ2], and using (2.4).

Proof of Lemma 5.4 We first recall some concepts introduced in [AJ1, Sec. 9]. 
Definition 5.1. We will say that the set $\left\{\theta_{1}, \ldots, \theta_{k+1}\right\}$ is $\epsilon$-uniform if

$$
\max _{z \in[-1,1] j=1, \ldots, k+1} \max _{\substack{\ell=1 \\ \ell \neq j}}^{k+1} \frac{\left.\mid z-\cos 2 \pi \theta_{\ell}\right) \mid}{\left.\mid \cos 2 \pi \theta_{j}-\cos 2 \pi \theta_{\ell}\right) \mid}<e^{k \epsilon} .
$$

$\epsilon$-uniformity (the smaller $\epsilon$ the better) involves uniformity along with certain cumulative repulsion of $\pm \theta_{i}(\bmod 1)$ 's.

Lemma 5.7. Let $\hat{\epsilon}>0$. If $\theta_{1}, \ldots, \theta_{k+1} \in A_{k,-\ln \lambda-\epsilon}$ and $k>k(\epsilon, \hat{\epsilon})$ is sufficiently large, then $\left\{\theta_{1}, \ldots, \theta_{k+1}\right\}$ is not $(\epsilon-\hat{\epsilon})$-uniform.

Proof. Write the polynomial $Q_{k}(z)$ in the Lagrange interpolation form using $\cos 2 \pi \theta_{1}$, $\ldots, \cos 2 \pi \theta_{k+1}$ :

$$
\left|Q_{k}(z)\right|=\left|\sum_{j=1}^{k+1} Q_{k}\left(\cos 2 \pi \theta_{j}\right) \frac{\prod_{\ell \neq j}\left(z-\cos 2 \pi \theta_{\ell}\right)}{\prod_{\ell \neq j}\left(\cos 2 \pi \theta_{j}-\cos 2 \pi \theta_{\ell}\right)}\right| .
$$

Let $\theta_{0}$ be such that $\left|P_{k}\left(\theta_{0}\right)\right| \geq \lambda^{-k}$. The lemma now follows immediately from 5.22 with $z=\cos 2 \pi\left(\theta_{0}+\frac{k-1}{2} \alpha\right)$.

We now define $I_{1}, I_{2} \subset \mathbb{Z}$ as follows:

(1) $I_{1}=\left[-2 s q_{n}+1,0\right]$ and $I_{2}=\left[k-2 s q_{n}+1, k+2 s q_{n}\right]$ if $k<\left|n_{j_{k}+1}\right| / 3$ and $n_{j_{k}} \geq 0$.

(2) $I_{1}=\left[1,2 s q_{n}\right]$ and $I_{2}=\left[k-2 s q_{n}+1, k+2 s q_{n}\right]$ if $k<\left|n_{j_{k}+1}\right| / 3$ and $n_{j_{k}}<0$.

(3) $I_{1}=\left[-2 s q_{n}+1,2 s q_{n}\right]$ and $I_{2}=\left[k-2 s q_{n}+1, k\right]$ if $\left|n_{j_{k}+1}\right| / 3 \leq k<\left|n_{j_{k}+1}\right| / 2$.

(4) $I_{1}=\left[-2 s q_{n}+1,2 s q_{n}\right]$ and $I_{2}=\left[k+1, k+2 s q_{n}\right]$ if $k \geq \mid n_{j_{k}+1 \mid} / 2$.

In either case, the total number of elements in $I_{1} \cup I_{2}$ is $6 s q_{n}$. Set $\theta_{j}=\theta+j \alpha$ for $j \in I_{1} \cup I_{2}$.

Lemma 5.8. If $\xi<C^{-1} \epsilon_{0}$ and $k>k\left(C_{0}, v, \xi\right)$ then the set $\left\{\theta_{j}\right\}_{j \in I_{1} \cup I_{2}}$ is $C \epsilon_{0}+C_{C_{0}} \xi$ uniform.

Proof. We will first estimate the numerator in 5.21). We have, in each case,

$$
\begin{aligned}
\sum_{\substack{j \in I_{1} \cup I_{2} \\
j \neq i}} \ln \left|\cos 2 \pi a-\cos 2 \pi \theta_{j}\right|= & \sum_{\substack{j \in I_{1} \cup I_{2} \\
j \neq i}} \ln \left|\sin 2 \pi \frac{a+\theta_{j}}{2}\right|+\sum_{\substack{j \in I_{1} \cup I_{2} \\
j \neq i}} \ln \left|\sin 2 \pi \frac{a-\theta_{j}}{2}\right| \\
& +\left(6 s q_{n}-1\right) \ln 2 \\
= & \Sigma_{+}+\Sigma_{-}+\left(6 s q_{n}-1\right) \ln 2
\end{aligned}
$$

Both $\Sigma_{+}$and $\Sigma_{-}$consist of $6 s$ terms of the form of 2.16 plus $6 s$ terms of the form

$$
\ln \min _{j=1, \ldots, q_{n-1}}|\sin 2 \pi(x+j \alpha / 2)|,
$$


minus $\ln \left|\sin \frac{a \pm \theta_{i}}{2}\right|$. Therefore, by 2.16

$$
\sum_{\substack{j \in I_{1} \cup I_{2} \\ j \neq i}} \ln \left|\cos 2 \pi a-\cos 2 \pi \theta_{j}\right| \leq-6 s q_{n} \ln 2+C s \ln q_{n} .
$$

To estimate the denominator of 5.21 we represent it again in the form 5.23 with $a=\theta_{i}$. Then

$$
\Sigma_{-}=\sum_{\substack{j \in I_{1} \cup I_{2} \\ j \neq i}} \ln |\sin \pi(i-j) \alpha| .
$$

$\Sigma_{-}$consists of $6 s$ terms of the form of 2.16 ) plus $6 s-1$ minimum terms (since when we split $\Sigma_{-}$into $6 s$ sums over intervals of length $q_{n}$ each, one of the sums will be exactly of the form of one of (2.16). For each $i, j \in I_{1} \cup I_{2}$, we have $|i-j| \leq k+4 s q_{n}<C_{C_{0}} s q_{n}$. In particular, $\ln |\sin \pi(i-j) \alpha| \geq-C_{C_{0}} s q_{n} \xi$ for large $q_{n}$ (depending on $v, \xi$ ). Notice that

$$
\max \{\ln |\sin x|, \ln |\sin (x+\pi j \alpha)|\}>-2 \xi q_{n}, \quad x \in \mathbb{R}, 0<|j|<q_{n+1},
$$

provided $q_{n}$ is sufficiently large (depending on $v$ and $\xi$ ). Using that $s q_{n}<q_{n+1}$ we get

$$
\Sigma_{-} \geq-6 s q_{n} \ln 2-C_{C_{0}} s q_{n} \xi
$$

Similarly, $\Sigma_{+}$consists of $6 s$ terms of the form of 2.16 plus $6 s$ minimum terms, each of the form

$$
\ln |\sin 2 \pi(\theta+(i+j) \alpha / 2)|
$$

for some $|j|<C_{C_{0}} s q_{n}<C_{C_{0}} q_{n+1}$, minus $\ln |\sin 2 \pi(\theta+i \alpha)|$ (which cancels a possible minimal term with $i=j$ ). Using (5.27), we see that at most 6 minimum terms are smaller than $-2 \xi q_{n}$. Let us estimate the smallest term with $j \neq i$.

Consider first cases (1) and (2) of the definition of $I_{1}, I_{2}$. Then, by the definition of $I_{i}$, $i=1,2$, and using (5.3) we have $i+j \neq n_{j_{k}}$ and $|i+j|<\left|n_{j_{k}+1}\right|$. Therefore the smallest term is bounded below by $-C \epsilon_{0} s q_{n}$ when $k>2\left|n_{j_{k}}\right|$, and by $-C_{C_{0}} \xi s q_{n}$ when $k \leq 2\left|n_{j_{k}}\right|$. In cases (3) and (4) we have $i+j \neq n_{j_{k}+1},|i+j|<2\left|n_{j_{k}+1}\right|$, and $s q_{n} \geq C_{C_{0}}^{-1}\left|n_{j_{k}+1}\right|$. Therefore the smallest term is bounded below by $-C \xi\left|n_{j_{k}+1}\right|>-C_{C_{0}} \xi s q_{n}$.

Putting all together, we see that

$$
\Sigma_{+} \geq-6 s q_{n} \ln 2-C s \ln q_{n}-\left(C \epsilon_{0}+C_{C_{0}} \xi\right) s q_{n}
$$

Combining (5.25), 5.28), and (5.30), we obtain

$$
\max _{j \in I_{1} \cup I_{2}} \prod_{\substack{\ell \in I_{1} \cup I_{2} \\ \ell \neq j}} \frac{\left|z-\cos 2 \pi \theta_{\ell}\right|}{\left|\cos 2 \pi \theta_{j}-\cos 2 \pi \theta_{\ell}\right|}<e^{\left(C \epsilon_{0}+C_{C_{0}} \xi\right) s q_{n}+C s \ln q_{n}}
$$

as desired. 
We can now finish the proof of Lemma 5.4 By Lemmas 5.7 and 5.8 at least one of $\theta_{j}, j \in I_{1} \cup I_{2}$, is not in $A_{6 s q_{n}-1,-\ln \lambda-\left(C_{C_{0}} \xi+C \epsilon_{0}\right)-\hat{\epsilon}}$ where $\hat{\epsilon}$ can be made arbitrarily small for large $n$. From (5.2) we see that the existence of a generalized eigenfunction $\hat{u}$ (as in Definition 3.1 implies that $y=0$ is $(m, k)$-singular for any $m>0$ and for $k$ sufficiently large depending on $m$. Thus by Lemma 5.3 with sufficiently small $\epsilon_{0}, \xi$ (depending also on $\left.C_{0}\right), y=0$ and $\delta<1 / 6$, we know that for all $j \in I_{1}, \theta_{j} \in A_{6 s q_{n}-1,-\ln \lambda-\left(C_{C_{0}} \xi+C \epsilon_{0}\right)-\hat{\epsilon}}$ for sufficiently large $n$. Let $j_{0} \in I_{2}$ be such that $\theta_{j_{0}} \notin A_{6 s q_{n}-1,-\ln \lambda-\left(C_{C_{0}} \xi+C \epsilon_{0}\right)-\hat{\epsilon}}$. Since $\left|j_{0}-k\right| \leq 2 s q_{n}$ we can apply again Lemma 5.3 with $\delta<1 / 6$ to conclude that $k$ is $\left(c, 6 s q_{n}-1\right)$-regular.

\section{Preliminary dynamical estimates}

\subsection{Lagrange interpolation argument}

Given Fourier coefficients $\hat{w}=\left(\hat{w}_{k}\right)_{k \in \mathbb{Z}}$ and an interval $I \subset \mathbb{Z}$, we let $w^{I}=$ $\sum_{k \in I} \hat{w}_{k} e^{2 \pi i k x}$. The length of the interval $I=[a, b]$ is $|I|=b-a$.

We will say that a trigonometric polynomial $p: \mathbb{R} / \mathbb{Z} \rightarrow \mathbb{C}$ has essential degree at most $k$ if its Fourier coefficients outside an interval $I$ of length $k$ are vanishing.

Theorem 6.1. Let $1 \leq r \leq\left[q_{n+1} / q_{n}\right]$. If $p$ has essential degree at most $k=r q_{n}-1$ and $x_{0} \in \mathbb{R} / \mathbb{Z}$ then for some absolute constant $C$,

$$
\|p\|_{0} \leq C q_{n+1}^{C r} \sup _{0 \leq j \leq k}\left|p\left(x_{0}+j \alpha\right)\right| .
$$

Proof. We may assume that $p(x)=P\left(e^{2 \pi i x}\right)$ where $P$ is a polynomial of degree $k$. Then by Lagrange interpolation,

$$
p(x)=\sum_{j=0}^{k} p\left(x_{0}+j \alpha\right) \prod_{\substack{0 \leq l \leq k \\ l \neq j}} \frac{e^{2 \pi i x}-e^{2 \pi i\left(x_{0}+l \alpha\right)}}{e^{2 \pi i\left(x_{0}+j \alpha\right)}-e^{2 \pi i\left(x_{0}+l \alpha\right)}} .
$$

Thus

$$
\ln \|p\|_{0} \leq \ln r q_{n}+\ln \sup _{0 \leq j \leq k}|p(x+j \alpha)|+\sup _{\substack{0 \leq j \leq k \\ x \in \mathbb{R}}} \sum_{\substack{0 \leq l \leq k \\ l \neq j}} \ln \frac{\left|1-e^{2 \pi i(x+l \alpha)}\right|}{\left|1-e^{2 \pi i(-j \alpha+l \alpha)}\right|} .
$$

In order to prove (6.1) it is enough to show that for $1 \leq s \leq r, 0 \leq j \leq r q_{n}-1$ and $x \in \mathbb{R}$ we have

$$
\begin{gathered}
\sum_{\substack{(s-1) q_{n} \leq l \leq s q_{n}-1 \\
l \neq j}} \ln \left|1-e^{2 \pi i(x+l \alpha)}\right| \leq C \ln q_{n}, \\
\sum_{\substack{(s-1) q_{n} \leq l \leq s q_{n}-1 \\
l \neq j}} \ln \left|1-e^{2 \pi i(-j \alpha+l \alpha)}\right| \geq-C \ln q_{n+1} .
\end{gathered}
$$


Consider first the case $(s-1) q_{n} \leq j \leq s q_{n}-1$. Then

$$
\sum_{(s-1) q_{n} \leq l \leq s q_{n}-1} \ln \left|1-e^{2 \pi i(x+l \alpha)}\right| \leq \sum_{\substack{(s-1) q_{n} \leq l \leq s q_{n}-1 \\ l \neq l_{0}}} \ln \left|1-e^{2 \pi i(x+l \alpha)}\right|,
$$

where $\left|1-e^{2 \pi i\left(x+l_{0} \alpha\right)}\right|$ is minimal. Using that $\left|1-e^{2 \pi i y}\right|=2|\sin \pi y|$ and Lemma 2.6 , we get 6.4). The same argument gives 6.5) even more directly, with $-C \ln q_{n}$ on the right hand side, since the sum to be estimated is already of the form considered in Lemma 2.6

Consider now the case $s-1 \neq\left[j / q_{n}\right]$. Writing

$$
\begin{aligned}
\sum_{\substack{(s-1) q_{n} \leq l \leq s q_{n}-1 \\
l \neq j}} \ln \left|1-e^{2 \pi i(x+l \alpha)}\right| & =\sum_{(s-1) q_{n} \leq l \leq s q_{n}-1} \ln \left|1-e^{2 \pi i(x+l \alpha)}\right|+\ln \left|1-e^{2 \pi i\left(x+l_{0} \alpha\right)}\right|,
\end{aligned}
$$

where $(s-1) q_{n} \leq l_{0} \leq s q_{n}-1$ is such that $\left|1-e^{2 \pi i\left(x+l_{0} \alpha\right)}\right|$ is minimal, we see that 6.4 follows from Lemma 2.6. To obtain 6.5 from Lemma 2.6, we must also show that

$$
\inf _{(s-1) q_{n} \leq l \leq s q_{n}-1} \ln \left|1-e^{2 \pi i(l-j) \alpha}\right| \geq-C \ln q_{n+1} .
$$

But this follows from 2.14 and 2.15. This concludes the proof of 6.1.

The Diophantine condition $\alpha \in \mathrm{DC}(\kappa, \tau)$ implies

$$
q_{n+1} \leq \kappa^{-1} q_{n}^{\tau-1} .
$$

In this case, 6.1 implies, with $c=\frac{1}{\tau-1}>0$ and $C=C(\kappa, \tau)$,

$$
\|p\|_{0} \leq C e^{C(1+k)^{1-c} \ln (1+k)} \sup _{0 \leq j \leq k}|p(x+j \alpha)|,
$$

since $1+k=r q_{n} \leq q_{n+1} \leq \kappa^{-1} q_{n}^{\tau-1}$.

\subsection{Polynomial growth}

From now on $\alpha \in \operatorname{DC}(\kappa, \tau)$ and the family $\left\{\hat{H}_{\lambda v, \alpha, \theta}\right\}_{\theta \in \mathbb{R}}$ is almost localized with parameters $\left(\epsilon_{0}, C_{0}, C_{1}, \epsilon_{1}\right)$. Let $E$ be in the spectrum and let $A=S_{\lambda v, E}$. Let $\theta=\theta(E)$ be given by Theorem 3.3. and let $\left\{n_{j}\right\}$ be the set of resonances of $\theta(E)$.

All constants may depend on $\kappa, \tau, C_{0}, \epsilon_{0}, \epsilon_{1}, C_{1}$, and on bounds on the analytic extension of $\lambda v$. We will use $C$ to denote large constants and $c$ to denote small constants. Further dependence on other parameters will be explicitly indicated, for instance, we will use $C_{\delta}$ for a large constant that depends on all parameters above and also on an arbitrary parameter $\delta>0$.

The specific values of generic constants such as $C, c, C_{\delta}$ may change through the argument, even when they appear in the same formula. In a few places we will use nongeneric constants, denoted $C^{(1)}, C^{(2)}, \ldots$, to simplify later referencing. 
Theorem 6.2. We have $L(\alpha, A)=0$.

Proof. Fixing a non-resonant $\theta^{\prime}$, Theorem 3.2 (see Remark 3.3) implies localization for $\hat{H}_{\lambda v, \alpha, \theta^{\prime}}$. By Theorem 2.5. $L\left(\alpha, S_{\lambda v, E^{\prime}}\right)=0$ for a dense set of $E^{\prime}$ in the spectrum. By continuity of the Lyapunov exponent [BJ2], this implies that $L\left(\alpha, S_{\lambda v, E}\right)=0$ for all $E$ in the spectrum 13

In our context (see Remark 2.1), this means that

$$
\sup _{x \in \mathbb{R} / \mathbb{Z}}\left\|A_{k}(x)\right\| \leq C_{\delta} e^{\delta k}, \quad \delta>0 .
$$

Our goal in this section is to improve subexponential growth to polynomial growth. This is of course still not optimal (see Corollary 4.5), but it is a good starting point.

Theorem 6.3. We have $\left\|A_{s}\right\|_{0} \leq C(1+s)^{C}, s \geq 0$.

We note that the proof of this estimate involves some themes which will appear again later.

Choose $4 C_{0}\left(\left|n_{j}\right|+1\right)<m<C_{0}^{-1}\left|n_{j+1}\right|$ of the form $m=r q_{k}-1<q_{k+1}$, let $I=[-[m / 2], m-[m / 2]]$ and define $u(x)=u^{I}(x)$. Let $U(x)=\left(\begin{array}{c}e^{2 \pi i \theta} u(x) \\ u(x-\alpha)\end{array}\right)$. Then

$$
A(x) \cdot U(x)-e^{2 \pi i \theta} U(x+\alpha)=e^{4 \pi i \theta}\left(\begin{array}{c}
h(x) \\
0
\end{array}\right)
$$

where

$$
\hat{h}_{k}=\chi_{I}(k) 2 \cos 2 \pi(\theta+k \alpha) \hat{u}_{k}+\sum \chi_{I}(k-j) \hat{v}_{j} \hat{u}_{k-j},
$$

where $\chi_{I}$ is the characteristic function of $I$. Since $\hat{H} \hat{u}=E \hat{u}$, we also have

$$
-\hat{h}_{k}=\chi_{\mathbb{Z} \backslash I}(k) 2 \cos 2 \pi(\theta+k \alpha) \hat{u}_{k}+\sum \chi_{\mathbb{Z} \backslash I}(k-j) \hat{v}_{j} \hat{u}_{k-j} .
$$

The estimates $\left|\hat{u}_{k}\right|<C_{1} e^{-\epsilon_{1}|k|}$ for $m / 4<|k|<m,\left|\hat{u}_{k}\right| \leq 1$ for all $k$ and $\left|\hat{v}_{k}\right| \leq C e^{-c|k|}$ for all $k$ then imply that $\left|\hat{h}_{k}\right| \leq C e^{-c m} e^{-c|k|}$, that is,

$$
\|h\|_{c} \leq C e^{-c m}
$$

Theorem 6.4. We have $\inf _{x \in \mathbb{R} / \mathbb{Z}}\|U(x)\| \geq c_{\delta} e^{-2 \delta m}, \delta>0, m \geq C$.

Proof. Otherwise, by 6.11, 6.12, 6.15, $|u(x+i \alpha)| \leq e^{-\delta m}$ for some $x \in \mathbb{R}$ and $0 \leq i \leq m$. Then, by Theorem 6.1. $\|u\|_{0} \leq C q_{k}^{C r} e^{-\delta m} \leq C_{\delta} e^{-\delta m / 2}$. This contradicts $\int u(x)=1$.

\footnotetext{
13 An alternative argument for zero Lyapunov exponent to be dense is the following. By duality [GJLS], if $\hat{H}$ has pure point spectrum for almost every $\theta$ then $H$ has absolutely continuous spectrum for almost every $\theta$ (see discussion in [BJ1] for the models considered here). By the Ishii-Pastur Theorem (e.g., $[\overline{\mathrm{CL}}]$ ), the Lyapunov exponent is zero densely in the spectrum.
} 
Let $B(x)$ be the matrix

$$
\left(\begin{array}{ll}
e^{2 \pi i \theta} u(x) & -\frac{1}{\|U(x)\|^{2}} \overline{u(x-\alpha)} \\
u(x-\alpha) & \frac{1}{\|U(x)\|^{2}} e^{-2 \pi i \theta} \overline{u(x)}
\end{array}\right) .
$$

By Theorem 6.4 and the trivial estimate $\|U\|_{0} \leq \mathrm{Cm}$ we have

$$
\|B\|_{0} \leq C_{\delta} e^{2 \delta m}, \quad \delta>0 .
$$

Thus

$$
B(x+\alpha)^{-1} A(x) B(x)=\left(\begin{array}{cc}
e^{2 \pi i \theta} & 0 \\
0 & e^{-2 \pi i \theta}
\end{array}\right)+\left(\begin{array}{cc}
\beta_{1}(x) & b(x) \\
\beta_{3}(x) & \beta_{4}(x)
\end{array}\right)
$$

with

$$
\left\|\left(\begin{array}{ll}
\beta_{1}(x) & b(x) \\
\beta_{3}(x) & \beta_{4}(x)
\end{array}\right)\right\|_{0} \leq C_{\delta} e^{4 \delta m} .
$$

Since the first column of $B$ satisfies 6.12 and $B \in \operatorname{SL}(2, \mathbb{C})$, 6.15) and 6.19) for appropriate $\delta$ give

$$
\left\|\beta_{1}\right\|_{0},\left\|\beta_{3}\right\|_{0},\left\|\beta_{4}\right\|_{0} \leq C e^{-c m} .
$$

Taking $\Phi(x)$ to be the product of $B(x)^{-1}$ and a constant diagonal matrix, $\Phi(x)=$ $D B(x)^{-1}$, where $D=\left(\begin{array}{cc}d & 0 \\ 0 & d^{-1}\end{array}\right)$ with $d^{2}=\max \left\{\left\|\beta_{3}\right\|_{0}^{1 / 2}, e^{-m}\right\}$, we get

$$
\Phi(x+\alpha) A(x) \Phi(x)^{-1}=\left(\begin{array}{cc}
e^{2 \pi i \theta} & 0 \\
0 & e^{-2 \pi i \theta}
\end{array}\right)+Q(x),
$$

where, by 6.20), 6.17, 6.19, , and (4.4) with appropriate $\delta,\|Q\|_{0} \leq C e^{-c m}$ and $\|\Phi\|_{0} \leq$ $C e^{m}$. This implies

$$
\left\|A_{s}\right\|_{0} \leq 2\|\Phi\|_{0}^{2} \leq C e^{C m}, \quad 0 \leq s \leq c^{(1)} e^{c^{(1)} m} .
$$

Proof of Theorem 6.3 Let $c^{(1)}$ be as in 6.22. For fixed $s$, let $m$ be minimal such that $s \leq c^{(1)} e^{c^{(1)} m}, 4 C_{0}\left(1+\left|n_{j}\right|\right)<m<C_{0}^{-1}\left|n_{j+1}\right|$, and $m=r q_{k}-1<q_{k+1}$ for some $j, k, r$. By Lemma 3.1. $m \leq C+C \ln (1+s)$. By 6.22, $\left\|A_{s}\right\|_{0} \leq C(1+s)^{C}$.

\subsection{Improved estimate on almost invariant sections}

In the previous argument, polynomial growth was obtained from an estimate on a suitably chosen "approximate Bloch wave". The first application of polynomial growth is that it allows us to deal, through a bootstrap argument, with much finer approximate Bloch waves. 
Lemma 6.5. Let $n=\left|n_{j}\right|+1<\infty$ and let $N=\left|n_{j+1}\right|$. Let $u(x)=u^{I}(x)$ for $I=$ $\left[-C_{0}^{-1} N+1, C_{0}^{-1} N-1\right]$. Define as before $U(x)=\left(\begin{array}{c}e^{2 \pi i \theta} u(x) \\ u(x-\alpha)\end{array}\right)$. Then we have the estimates

$$
\begin{gathered}
A(x) \cdot U(x)=e^{2 \pi i \theta} U(x+\alpha)+\left(\begin{array}{c}
h(x) \\
0
\end{array}\right) \quad \text { with } \quad\|h\|_{c} \leq e^{-c N}, \\
\inf _{|\Im z|<c n^{-C}}\|U(x)\| \geq c n^{-C}, \quad N \geq C, \\
\|U\|_{0} \leq C n .
\end{gathered}
$$

Proof. Estimate 6.23 is obtained exactly as 6.15. For 6.24, let $4 C_{0} n<m<C_{0}^{-1} N$ be of the form $m=q_{k}-1$, and let $J=[-[m / 2], m-[m / 2]]$. Define

$$
U^{J}(x)=\left(\begin{array}{c}
e^{2 \pi i \theta} u^{J}(x) \\
u^{J}(x-\alpha)
\end{array}\right) .
$$

Theorem 3.2 implies

$$
\left\|U-U^{J}\right\|_{c} \leq C e^{-c m} .
$$

Arguing as in Theorem 6.4 we get

$$
\inf _{x \in \mathbb{R} / \mathbb{Z}}\left\|U^{J}(x)\right\| \geq c m^{-C}, \quad m \geq C
$$

(the estimate is better since $|J|=q_{k}-1$, and because we can use Theorem 6.3 instead of 6.11). Obvious bounds on the derivative of $U^{J}$ then give $\left\|\frac{d}{d z} U^{J}(z)\right\|_{\mathrm{cm}^{-1}} \leq \mathrm{Cm}^{2}$, which together with 6.26) and 6.27) implies

$$
\inf _{|\Im z|<c m^{-C}}\|U(z)\| \geq c m^{-C}, \quad m \geq C^{(1)} .
$$

By Lemma 3.1 and the Diophantine condition, if $m>\max \left\{4 C_{0} n, C^{(1)}\right\}$ is minimal of the form $m=q_{k}-1$ then $m<C n^{C}<C_{0}^{-1} N, N \geq C$. Together with 6.28, this gives 6.24.

Estimate 6.25 is immediate by Theorem 3.2 .

\section{7. (Complex) almost triangularization: proof of Theorem 3.4}

Let $U(x)$ be as in Lemma 6.5. Let $B(x)$ be defined by 6.16. By Lemma 6.5 we get the bound

$$
\|B\|_{c n^{-C}} \leq C n^{C} .
$$


Here the complex extension of $B$ is the holomorphic one (not the one given by (6.16). By the same computation as in (6.18) and using Lemma 6.5 we get the improved estimate

$$
B(x+\alpha)^{-1} A(x) B(x)=\left(\begin{array}{cc}
e^{2 \pi i \theta} & 0 \\
0 & e^{-2 \pi i \theta}
\end{array}\right)+\left(\begin{array}{cc}
\beta_{1}(x) & b(x) \\
\beta_{3}(x) & \beta_{4}(x)
\end{array}\right)
$$

where $\|b\|_{c n^{-C}} \leq C n^{C}$ and $\left\|\beta_{1}\right\|_{c n^{-C}},\left\|\beta_{3}\right\|_{c n^{-C}},\left\|\beta_{4}\right\|_{c n^{-C}} \leq C e^{-c N}$. Thus the Fourier coefficients of $b$ satisfy the estimate

$$
\left|\hat{b}_{k}\right| \leq C n^{C} e^{-c n^{-C}|k|}
$$

Notice that the estimates so far cover the case of the first resonance $(n=1)$, so we will assume from now on that $n>1$.

In the estimates to follow, we shift one scale back. So take $\underline{n}=1+\left|n_{j-1}\right|, \underline{N}=\left|n_{j}\right|$, and the remaining notation is clear. Split $\underline{b}(x)=\underline{b}^{l}(x)+\underline{b}^{h}(x)$ where the first term has the (low frequency) Fourier coefficients with $|k|<\underline{N}$ and $\underline{b}^{h}$ is the rest. We solve exactly

$$
\left(\begin{array}{cc}
1 & -\underline{\phi}(x+\alpha) \\
0 & 1
\end{array}\right)\left(\begin{array}{cc}
e^{2 \pi i \theta} & \underline{b}^{l}(x) \\
0 & e^{-2 \pi i \theta}
\end{array}\right)\left(\begin{array}{cc}
1 & \underline{\phi}(x) \\
0 & 1
\end{array}\right)=\left(\begin{array}{cc}
e^{2 \pi i \theta} & 0 \\
0 & e^{-2 \pi i \theta}
\end{array}\right) .
$$

This corresponds to solving

$$
\underline{b}^{l}(x)-e^{-2 \pi i \theta} \underline{\phi}(x+\alpha)+e^{2 \pi i \theta} \underline{\phi}(x)=0,
$$

or in terms of Fourier coefficients,

$$
\underline{\hat{\phi}}_{k}=-\underline{\hat{b}}_{k} \frac{e^{-2 \pi i \theta}}{1-e^{-2 \pi i(2 \theta-k \alpha)}}
$$

for $|k|<\underline{N}$ and $\underline{\hat{\phi}}_{k}=0$ for $|k| \geq \underline{N}$. By $\sqrt{7.3}$, and since $\alpha \in \mathrm{DC}(\kappa, \tau)$ and $|k|<\underline{N}$, we have $\|\underline{\phi}(x)\|_{c \underline{n}^{-C}} \leq C \underline{N}^{C}$. We conclude that with

$$
\underline{\Phi}(x)=\left(\begin{array}{cc}
1 & -\underline{\phi}(x) \\
0 & 1
\end{array}\right) \underline{B}(x)^{-1}
$$

we have

$$
\underline{\Phi}(x+\alpha) A(x) \underline{\Phi}(x)^{-1}=\left(\begin{array}{cc}
e^{2 \pi i \theta} & 0 \\
0 & e^{-2 \pi i \theta}
\end{array}\right)+\underline{\Psi}(x)
$$

where

$$
\underline{\Psi}(x)=\left(\begin{array}{cc}
1 & -\underline{\phi}(x+\alpha) \\
0 & 1
\end{array}\right)\left(\begin{array}{ll}
\underline{\beta}_{1}(x) & \underline{b}^{h}(x) \\
\underline{\beta}_{3}(x) & \underline{\beta}_{4}(x)
\end{array}\right)\left(\begin{array}{cc}
1 & \phi(x) \\
0 & 1
\end{array}\right)
$$

and $\|\underline{\Phi}\|_{c \underline{n}^{-C}} \leq C \underline{N}^{C}$. Since, by $7.3,\left\|\underline{b}^{h}\right\|_{c \underline{n}^{-C}} \leq C \underline{n}^{C} e^{-c \underline{n}^{-C} \underline{N}} \leq C e^{-c \underline{n}^{-C}} \underline{N}$, we also have $\|\underline{\Psi}\|_{c \underline{n}^{-C}} \leq C e^{-c \underline{n}^{-C}} \underline{N}$. It follows that

$$
\left\|A_{s}\right\|_{c \underline{n}^{-C}} \leq C \underline{N}^{C}, \quad 0 \leq s \leq c e^{c \underline{n}^{-C}} \underline{N} .
$$


Back to the original scale, we get, using Lemma 3.1 .

$$
\left\|A_{s}\right\|_{c(\ln n)^{-C}} \leq C n^{C}, \quad 0 \leq s \leq c e^{c n(\ln n)^{-C}} .
$$

Let $\Delta \geq n$ be minimal so that $\left|\hat{b}_{k}\right| \leq \Delta e^{-\Delta^{-1}|k|}, k \in \mathbb{Z}$. Let us consider a different decomposition $b=b^{r}+b^{l}+b^{h}$ so that now $b^{r}$ has only the (resonant) $n_{j}$-th Fourier coefficient, $b^{l}$ has the (low frequency) Fourier coefficients with $|k| \leq \Delta^{3}$ (except for $n_{j}$ ) and $b^{h}$ has the (high frequency) Fourier coefficients with $|k|>\Delta^{3}$. By $7.3, \Delta \leq C n^{C}$, and the definition of $\Delta$ gives

$$
\left\|b^{h}\right\|_{c n^{-C}} \leq C e^{-n}
$$

We can solve

$$
\left(\begin{array}{cc}
1 & -\phi(x+\alpha) \\
0 & 1
\end{array}\right)\left(\begin{array}{cc}
e^{2 \pi i \theta} & b^{l}(x) \\
0 & e^{-2 \pi i \theta}
\end{array}\right)\left(\begin{array}{cc}
1 & \phi(x) \\
0 & 1
\end{array}\right)=\left(\begin{array}{cc}
e^{2 \pi i \theta} & 0 \\
0 & e^{-2 \pi i \theta}
\end{array}\right) .
$$

As in 7.6 it follows that $\|\phi(x)\|_{c n^{-C}} \leq C n^{C}$. We conclude that the matrix $\Phi(x)=$ $\left(\begin{array}{cc}1 & -\phi(x) \\ 0 & 1\end{array}\right) B(x)^{-1}$ satisfies

$$
\Phi(x+\alpha) A(x) \Phi(x)^{-1}=\left(\begin{array}{cc}
e^{2 \pi i \theta} & 0 \\
0 & e^{-2 \pi i \theta}
\end{array}\right)+\left(\begin{array}{cc}
0 & b^{r}(x)+b^{h}(x) \\
0 & 0
\end{array}\right)+\Psi(x)
$$

with

$$
\|\Phi\|_{c n^{-C}} \leq C n^{C} \quad \text { and } \quad\|\Psi\|_{c n^{-C}} \leq C e^{-c N} .
$$

We now estimate $b^{r}(x)=\hat{b}_{n_{j}} e^{2 \pi i n_{j} x}$. Let

$$
W(x)=\left(\begin{array}{cc}
e^{2 \pi i \theta} & b^{r}(x) \\
0 & e^{-2 \pi i \theta}
\end{array}\right) .
$$

We can compute exactly

$$
W_{s}=\left(\begin{array}{cc}
e^{2 \pi i s \theta} & b^{s}(x) \\
0 & e^{-2 \pi i s \theta}
\end{array}\right)
$$

with

$$
\left|b^{s}(x)\right|=\left|\hat{b}_{n_{j}} \sum_{k=0}^{s-1} e^{-2 \pi i k\left(2 \theta-n_{j} \alpha\right)}\right|=\left|\hat{b}_{n_{j}} \frac{\sin \pi s\left(2 \theta-n_{j} \alpha\right)}{\sin \pi\left(2 \theta-n_{j} \alpha\right)}\right| \quad \text { if } \sin \pi\left(2 \theta-n_{j} \alpha\right) \neq 0
$$

and $\left|b^{s}(x)\right|=s\left|\hat{b}_{n_{j}}\right|$ otherwise. Therefore we have

$$
\left\|W_{s}\right\|_{0} \geq s\left|\hat{b}_{n_{j}}\right| / 10, \quad 0 \leq s \leq\left\|2 \theta-n_{j} \alpha\right\|_{\mathbb{R} / \mathbb{Z}}^{-1} / 10 .
$$

On the other hand, by 7.3 ,

$$
\left\|W_{s}\right\|_{0} \leq 1+s\left|\hat{b}_{n_{j}}\right| \leq C(1+s) n^{C}, \quad s \geq 0 .
$$


Using 7.13, we get $A=\Phi(x+\alpha)^{-1}(W(x)+Z(x)) \Phi(x)$, so that

$$
\left\|A_{s}\right\|_{0} \geq\|\Phi\|_{0}^{-2}\left(\left\|W_{s}\right\|_{0}-\sum_{k=1}^{s}\left(\begin{array}{l}
s \\
k
\end{array}\right)\|Z\|_{0}^{k} \max _{0 \leq j<s}\left\|W_{j}\right\|_{0}^{1+k}\right) .
$$

By (7.14) and 7.11 we have $\|Z\|_{0} \leq C e^{-n}$. Thus, 7.18 and 7.19 imply

$$
\left\|A_{s}\right\|_{0} \geq c n^{-C}\left(\left\|W_{s}\right\|_{0}-C e^{-c n}\right), \quad 0 \leq s \leq c e^{c n} .
$$

By 7.10 and 7.20), $\left\|W_{s}\right\|_{0} \leq C n^{C}, 0 \leq s \leq c e^{c n(\ln n)^{-C}}$. Together with Lemma 3.1 and (7.17), we get the estimate

$$
\left|\hat{b}_{n_{j}}\right| \leq C n^{C} e^{-c n(\ln n)^{-C}} \leq C e^{-c n(\ln n)^{-C}} .
$$

The result follows from (7.13, 7.14, (7.11) and 7.21).

\section{8. (Real) almost conjugacy to rotations: proof of Theorem 3.5}

Let $U(x)$ be as in Lemma 6.5 . Let $B(x)$ be the matrix with columns $U(x)$ and $\overline{U(x)}$ on

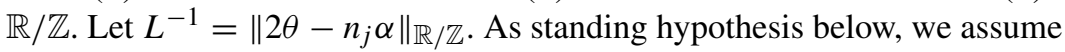

$$
0<L^{-1}<c
$$

Lemma 8.1. For any $\epsilon>0$, we have

$$
\inf _{x \in \mathbb{R} / \mathbb{Z}}|\operatorname{det} B(x)| \geq c L^{-C} .
$$

Proof. Recall that for any $2 \times 2$ complex matrix $M$ with columns $V$ and $W$,

$$
|\operatorname{det} M|=\|V\| \min _{\lambda \in \mathbb{C}}\|W-\lambda V\|
$$

Notice that the minimizing $\lambda$ satisfies $\|\lambda V\| \leq\|W\|$.

Minimize over $\lambda \in \mathbb{C}$ and $x \in \mathbb{R} / \mathbb{Z}$ the quantity $\left\|e^{-\pi i n_{j} x} \overline{U(x)}-\lambda e^{\pi i n_{j} x} U(x)\right\|$. This gives some $\lambda_{0}$ and $x_{0}$. If the estimate does not hold then, using Lemma 6.5, Theorem 6.3. (8.3), and Lemma 3.1, we would have

$$
\left\|e^{-2 \pi i s \theta} e^{-\pi i n_{j} x_{0}} \overline{U\left(x_{0}+s \alpha\right)}-e^{2 \pi i s \theta} e^{\pi i n_{j} x_{0}} \lambda_{0} U\left(x_{0}+s \alpha\right)\right\| \leq L^{-1}, \quad 0 \leq s \leq L .
$$

This implies that $\left\|e^{-\pi i n_{j}\left(x_{0}+s \alpha\right)} \overline{U\left(x_{0}+s \alpha\right)}-\lambda_{0} e^{\pi i n_{j}\left(x_{0}+s \alpha\right)} U\left(x_{0}+s \alpha\right)\right\| \leq L^{-1}+$ $C s L^{-1}\|U\|_{0} \leq L^{-1 / 3}$ for $0 \leq s \leq L^{1 / 3}$ (recalling 6.25 ).

As in the proof of Lemma 6.5 , let $m>(\ln L)^{2}>4 C_{0} n$ (here the second inequality is a consequence of (8.1) be minimal of the form $m=q_{k}-1$, let $J=[-[m / 2], m-$ $[m / 2]]$ and let $U^{J}(x)=\left(\begin{array}{c}e^{2 \pi i \theta} u^{J}(x) \\ u^{J}(x-\alpha)\end{array}\right)$. By Lemma 3.1 and the Diophantine condition, $m<C(\ln L)^{C}<C_{0}^{-1} N$. Theorem 3.2 then gives $\left\|U-U^{J}\right\|_{c} \leq L^{-1}$. Using Theorems 
6.1 and 6.3 we obtain $\left\|e^{-\pi i n_{j} x} \overline{U^{J}(x)}-e^{\pi i n_{j} x} \lambda_{0} U^{J}(x)\right\|_{0} \leq C(\ln L)^{C}\left(L^{-1 / 3}+2 L^{-1}\right)$. Thus

$$
\sup _{x \in \mathbb{R} / \mathbb{Z}}\left\|e^{-\pi i n_{j} x} \overline{U(x)}-e^{\pi i n_{j} x} \lambda_{0} U(x)\right\| \leq L^{-1 / 4} .
$$

Substituting $x_{1}=x_{0}+s \alpha$ in $(8.4)$ and taking $s=[L / 2]$, we get $\| i e^{-\pi i n_{j} x_{1}} \overline{U\left(x_{1}\right)}+$ $i e^{\pi i n_{j} x_{1}} \lambda_{0} U\left(x_{1}\right)\left\|\leq L^{-1}+C L^{-1}\right\| U \|_{0}$, so that, by $(8.5)$ and (6.25), $\left\|U\left(x_{1}\right)\right\| \leq L^{-1 / 5}$. However, 6.24 and Lemma 3.1 imply $\left\|U\left(x_{1}\right)\right\| \geq c(\ln L)^{-C}$, giving a contradiction.

Lemma 8.2. Let $x_{0} \in \mathbb{R} / \mathbb{Z}$. Then

$$
\sup _{|\Im z|<c}\left|\operatorname{det} B(z)-\operatorname{det} B\left(x_{0}\right)\right| \leq C e^{-c N} .
$$

Proof. By 6.23,

$$
A(x) B(x)=\left(\begin{array}{cc}
e^{2 \pi i \theta} & 0 \\
0 & e^{-2 \pi i \theta}
\end{array}\right) B(x+\alpha)+\left(\begin{array}{c}
h(x) \\
0
\end{array}\right), \quad\|h\|_{c} \leq e^{-c N},
$$

thus $\left|\operatorname{det} B\left(x_{0}+\alpha\right)-\operatorname{det} B\left(x_{0}\right)\right| \leq C e^{-c N}$. This gives $\left|\operatorname{det} B\left(x_{0}+k \alpha\right)-\operatorname{det} B\left(x_{0}\right)\right| \leq$ $C e^{-c N}$ for $0 \leq k \leq 4 N$. The function $x \mapsto \operatorname{det} B\left(x_{0}+k \alpha\right)-\operatorname{det} B(x)$ is a trigonometric polynomial of essential degree bounded by $4 N$. By Theorem 6.1] $\left|\operatorname{det} B(x)-\operatorname{det} B\left(x_{0}\right)\right| \leq$ $C e^{-c N}, x \in \mathbb{R} / \mathbb{Z}$. By Theorem 3.2. $\|U(z)\|_{c} \leq C e^{C n}{ }^{14}$ so $z \mapsto \operatorname{det} B(z)-\operatorname{det} B\left(x_{0}\right)$ is bounded by $C e^{C n}$ over $|\Im z|<c^{(2)}$. By the Hadamard three-circle theorem,

$\ln \sup _{|\Im z|=\delta c^{(2)}}\left|\operatorname{det} B(z)-\operatorname{det} B\left(x_{0}\right)\right| \leq(1-\delta) \ln \sup _{|\Im z|=0}+\delta \ln \sup _{|\Im z|=c^{(2)}} \leq-c N, \quad 0 \leq \delta<c$.

Thus $\left|\operatorname{det} B(z)-\operatorname{det} B\left(x_{0}\right)\right| \leq C e^{-c N}$ for $|\Im z|<c$.

Theorem 8.3. We have

$$
\inf _{|\Im z|<c}|\Im \operatorname{det} B(z)| \geq c L^{-C} \geq C / N^{C} .
$$

Proof. Notice that $\Re \operatorname{det} B(x)=0$ for $x \in \mathbb{R} / \mathbb{Z}$. The result now follows from 8.2 and 8.6. (taking into account Lemma 3.1).

Remark 8.1. Optimizing the method (and using other estimates obtained in this paper) gives $|\operatorname{det} B(z)| \geq c_{\delta} L^{-1-\delta}$ for $|\Im z|<c$. This will be explored and used in [AJ2].

Take now $S=\Re U, T=\Im U$ on $\mathbb{R} / \mathbb{Z}$. Then $B=\left(\begin{array}{ll}S & \pm T\end{array}\right)\left(\begin{array}{cc}1 & 1 \\ \pm i & \mp\end{array}\right)$. Let $W_{1}$ be the matrix with columns $S$ and $\pm T$, so as to have $\operatorname{det} W_{1}>0$. Since

$$
\left(\begin{array}{cc}
1 & 1 \\
\pm i & \mp i
\end{array}\right)\left(\begin{array}{cc}
e^{2 \pi i \theta} & 0 \\
0 & e^{-2 \pi i \theta}
\end{array}\right)\left(\begin{array}{cc}
1 & 1 \\
\pm i & \mp i
\end{array}\right)^{-1}=R_{\mp \theta}
$$

14 Actually, even a trivial bound like $\|U(z)\|_{c} \leq C e^{C N}$ would do here. 
by (6.23) we have

$$
\left\|A(x) \cdot W_{1}(x)-W_{1}(x+\alpha) \cdot R_{\mp \theta}\right\| \leq C e^{-c N}, \quad|\Im x|<c
$$

(the complex extension considered here is the holomorphic one). We define $W(x)=$ $|\operatorname{det} B(x) / 2|^{-1 / 2} W_{1}(x)$ on $\mathbb{R} / \mathbb{Z}$, so that $\operatorname{det} W=1$ (by Theorem 8.3 there is no problem with branching when extending $|\operatorname{det} B(x)|^{-1 / 2}$ to $\left.|\Im x|<c\right)$. Then

$$
\left\|A(x) \cdot W(x)-\frac{|\operatorname{det} B(x+\alpha)|^{1 / 2}}{|\operatorname{det} B(x)|^{1 / 2}} W(x+\alpha) \cdot R_{\mp \theta}\right\| \leq C e^{-c N}, \quad|\Im x|<c .
$$

A combination of 8.6 and Theorem 8.3 gives

$$
\left|\frac{|\operatorname{det} B(x+\alpha)|^{1 / 2}}{|\operatorname{det} B(x)|^{1 / 2}}-1\right| \leq C e^{-c N}, \quad|\Im x|<c .
$$

Also, using Theorems 3.2 and 8.3 we get $\|W\|_{c} \leq C N^{C} e^{c n}$. Thus

$$
\left\|A(x) \cdot W(x)-W(x+\alpha) \cdot R_{\mp \theta}\right\| \leq C e^{-c N}, \quad|\Im x|<c .
$$

To conclude, we need to show that $|\operatorname{deg} W| \leq C n$. Set $\tilde{U}(x)=e^{\pi i n_{j} x} U(x)$, and $\tilde{S}=\Re \tilde{U}, \tilde{T}=\Im \tilde{U}, \tilde{W}_{1}=(\tilde{S} \pm \tilde{T}), \tilde{W}(x)=|\operatorname{det} B(x) / 2|^{1 / 2} \tilde{W}_{1}(x)$ on $\mathbb{R} / 2 \mathbb{Z}$. Then $\tilde{W}(x)=W(x) R_{\mp n_{j} x / 2}$, so $\operatorname{deg} \tilde{W}-2 \operatorname{deg} W=\mp n_{j}$, where $\operatorname{deg} \tilde{W}$ is the degree of $\tilde{W}: \mathbb{R} / 2 \mathbb{Z} \rightarrow \operatorname{SL}(2, \mathbb{R})$ and $\operatorname{deg} W$ is understood in the usual sense, as the degree of $W: \mathbb{R} / \mathbb{Z} \rightarrow \operatorname{SL}(2, \mathbb{R})$.

The degree of $\tilde{W}: \mathbb{R} / 2 \mathbb{Z} \rightarrow \operatorname{SL}(2, \mathbb{R})$ is the same as the degree of any of its columns, considered as maps $\mathbb{R} / 2 \mathbb{Z} \rightarrow \mathbb{R}^{2} \backslash\{0\}$. Thus we need to show that the degree of $M$ : $\mathbb{R} / 2 \mathbb{Z} \rightarrow \mathbb{R}^{2} \backslash\{0\}$, for either $M=\tilde{S}$ or $M=\tilde{T}$, satisfies $|\operatorname{deg} M| \leq C n$.

Notice that

$$
\left\|\int_{\mathbb{R} / 2 \mathbb{Z}} e^{-\pi i n_{j} x}(\tilde{S}(x)+i \tilde{T}(x)) d x\right\|=\left\|\int_{\mathbb{R} / 2 \mathbb{Z}} U(x) d x\right\| \geq 2,
$$

since $\hat{u}_{0}=1$. Select $M(x)=\tilde{S}(x)$ or $M(x)=\tilde{T}(x)$ so that $\int_{\mathbb{R} / 2 \mathbb{Z}}\|M(x)\| \geq 1$. Then, by 6.23, for $|\Im x|<c$ we have $\left\|A(x) \cdot \tilde{U}(x)-e^{\pi i\left(2 \theta-n_{j} \alpha\right)} \tilde{U}(x+\alpha)\right\|_{0} \leq C e^{-c N}$. By Lemma 3.1 and using 6.25 ) and $L^{-1} \leq e^{-\epsilon_{0}|n|}$, we have $|\|A(x) \cdot M(x)\|-\|M(x+\alpha)\|| \leq$ $C L^{-1} n \leq C L^{-c}$ for $x \in \mathbb{R} / 2 \mathbb{Z}$. Using Theorem 6.3, we obtain, arguing as in the proof of Lemma 6.5 .

$$
\inf _{|\Im x|<c n^{-C}}\|M(x)\| \geq c n^{-C} .
$$

This already gives the bound $|\operatorname{deg} M| \leq C n^{C}$ by the obvious derivative estimate. To obtain a linear bound, let $\tilde{M}(x)$ be a vector obtained by cutting off the Fourier modes of $M(x)$ with $|k / 2|>\Delta$, where $\Delta$ is chosen minimal so that $\|\tilde{M}(x)-M(x)\|<\|M(x)\|$, $x \in \mathbb{R} / 2 \mathbb{Z}$. Then by Theorem 3.2 and 8.14$], \Delta<C n$. By Rouché's Theorem, the degree of $M$ is the same as the degree of $\tilde{M}$. Consider now a coordinate of $x \mapsto \tilde{M}(2 x)$ which is not identically vanishing. It is a trigonometric polynomial of essential degree at most $C n$, and since it is not identically vanishing, it has at most $C n$ zeroes in $\mathbb{R} / \mathbb{Z}$. It follows that $|\operatorname{deg} \tilde{M}|$ (and thus $|\operatorname{deg} M|)$ is bounded by $C n$, and we conclude that $|\operatorname{deg} W| \leq C n$. 


\section{Appendix. The perturbative theory of Eliasson}

The following result is due to Hakan Eliasson.

Theorem A.1. Let $\alpha \in \mathrm{DC}(\kappa, \tau)$, and let $A: \mathbb{R} / \mathbb{Z} \rightarrow \operatorname{SL}(2, \mathbb{R})$ be analytic. Assume that $\left\|A-A_{*}\right\|_{\epsilon}<C_{0}^{-1} \epsilon^{r_{0}}$ where $A_{*} \in \operatorname{SL}(2, \mathbb{R}), 0<\epsilon<1, r_{0}=r_{0}(\tau)$ and $C_{0}=$ $C_{0}\left(\kappa, \tau,\left\|A_{*}\right\|\right)$. Then

(1) If $\rho(\alpha, A)$ is either Diophantine or rational with respect to $\alpha$ then $(\alpha, A)$ is analytically reducible.

(2) If $(\alpha, A)$ is not hyperbolic then $\sup k^{-1}\left\|A_{k}\right\|_{0} \leq C_{0}$.

(3) If $\rho(\alpha, A)$ is not rational then $\lim k^{-1}\left\|A_{k}\right\|_{0}=0$.

Actually, what is considered in $[\mathrm{E}]$ is the case of continuous time, and for cocycles of Schrödinger type (he also treats the case of several frequencies). The considerations for discrete time are similar and are carried out in the thesis of his student Sana Ben Hadj Amor $[\overline{\mathrm{Am}}]$. In [E], Eliasson goes on to establish absolutely continuous spectrum for all phases for the associated Schrödinger operators, while the proof of 1/2-Hölder continuity of the integrated density of states is the main result of Amor's thesis.

The KAM scheme of Eliasson has been extended to the $C^{\infty}$ case [AK2]. This generalization motivated the introduction of the concept of "almost reducibility" in the $C^{\infty}$ case.

\section{A.1. Quick reduction to the perturbative regime}

Reduction to the perturbative regime of Eliasson is a much less subtle result than showing almost reducibility. It can be concluded from almost localization just after $\$ 6$, since it is clearly 15 implied by the following result.

Theorem A.2. If $\theta$ is resonant, there exists arbitrarily small $\epsilon>0,-1 \leq \kappa \leq 1$, and $B: \mathbb{R} / \mathbb{Z} \rightarrow \operatorname{PSL}(2, \mathbb{R})$ analytic such that $\|B\|_{\epsilon} \leq C \epsilon^{-C}$ and

$$
\left\|B(x+\alpha) A(x) B(x)^{-1}-A_{*}\right\|_{\epsilon} \leq C e^{-c / \epsilon^{c}}, \quad \text { where } \quad A_{*}=\left(\begin{array}{cc}
1 & \kappa \\
0 & 1
\end{array}\right) .
$$

Proof. The beginning of the proof coincides with a small part of the argument used in the proof of Theorem 3.5 but we briefly repeat it here for the readers' convenience. Let $n=\left|n_{j}\right|+1, N=\left|n_{j+1}\right|$, and $I=\left[-C_{0}^{-1} N+1, C_{0}^{-1} N-1\right]$. Let $u=u^{I}$, and define $U(x)=\left(\begin{array}{c}e^{2 \pi i \theta} u(x) \\ u(x-\alpha)\end{array}\right)$ as usual. Let $\tilde{U}(x)=e^{\pi i n_{j} x} U(x)$ and $\tilde{S}(x)=\Re \tilde{U}(x), \tilde{T}(x)=$ $\Im \tilde{U}(x)$. Since 8.13 holds, we can choose $M=\tilde{S}$ or $M=\tilde{T}$ so that $\int_{\mathbb{R} / 2 \mathbb{Z}}\|M(x)\| \geq 1$. Notice that by Lemma 6.5 and the definition of resonance,

$$
A(x) \cdot M(x)=M(x+\alpha)+O\left(e^{-c n}\right), \quad|\Im x|<c .
$$

\footnotetext{
15 Recall that if $\theta$ is non-resonant then by Theorems 2.5 and $3.2,(\alpha, A)$ is conjugate to a constant cocycle (which is obviously in Eliasson's perturbative regime).
} 
Then, arguing as in Lemma 6.5, we have (8.14). Let $W$ be the matrix with columns $M$ and $\|M\|^{-2} R_{1 / 4} M$. Then by (A.1) and (8.14) we have

$$
W(x+\alpha)^{-1} A(x) W(x)=\left(\begin{array}{ll}
1 & 0 \\
0 & 1
\end{array}\right)+\left(\begin{array}{ll}
\beta_{1}(x) & \beta_{2}(x) \\
\beta_{3}(x) & \beta_{4}(x)
\end{array}\right)
$$

with $\left\|\beta_{1}\right\|_{c n^{-C}},\left\|\beta_{3}\right\|_{c n^{-C}},\left\|\beta_{4}\right\|_{c n^{-C}} \leq C e^{-c n}$ and $\left\|\beta_{2}\right\|_{c n^{-C}} \leq C n^{C}$. Solve the cohomological equation $\phi(x+\alpha)+\beta_{2}(x)-\phi(x)=\int_{0}^{2} \beta_{2}(x) d x / 2=b$, with $\int_{0}^{2} \phi(x) d x=0$. Then, by writing out the Fourier coefficients and since $\alpha \in$ DC we get $\|\phi\|_{\mathrm{Cn}^{-C}} \leq$

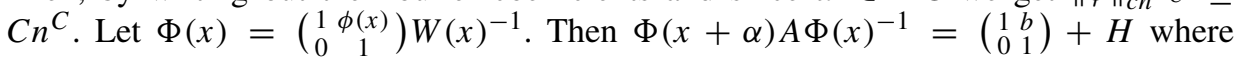
$\|H\|_{c n^{-C}} \leq C e^{-c n}$. Let $d^{2}=\min \left\{|b|^{-1}, 1\right\}$ and let $D=\left(\begin{array}{cc}d & 0 \\ 0 & d^{-1}\end{array}\right)$. Then $B(x)=$ $D \Phi(x+\alpha) A(x) \Phi(x)^{-1} D^{-1}$ is such that

$$
\left\|B-\left(\begin{array}{cc}
1 & \kappa \\
0 & 1
\end{array}\right)\right\|_{c n^{-C}} \leq C e^{-c n}
$$

for some $-1 \leq \kappa \leq 1$.

Acknowledgments. This work was supported in part by NSF, grant DMS-0300974 and DMS0601081, and BSF, grant 2006483. This research was partially conducted during the period when A.A. served as a Clay Research Fellow.

\section{References}

[Am] Amor, S.: Hölder continuity of the rotation number for quasi-periodic co-cycles in $\mathrm{SL}(2, \mathbb{R})$. Comm. Math. Phys. 287, 565-588 (2009)

[AA] Aubry, S., André, G.: Analyticity breaking and Anderson localization in incommensurate lattices. In: Group Theoretical Methods in Physics (Kiryat Anavim, 1979), Ann. Israel Phys. Soc. 3, Hilger, Bristol, 133-164 (1980) Zbl 0943.82510 MR 0626837

[A] Avila, A.: The absolutely continuous spectrum of the almost Mathieu operator. Preprint

[AD] Avila, A., Damanik, D.: Absolute continuity of the integrated density of states for the almost Mathieu operator with non-critical coupling. Invent. Math. 172, 439-453 (2008) Zbl 1149.47021 MR 2390290

[AJ1] Avila, A., Jitomirskaya, S.: The Ten Martini Problem. Ann. of Math. 170, 303-342 (2009) MR 2521117

[AJ2] Avila, A., Jitomirskaya, S.: Hölder continuity of absolutely continuous spectral measures for one-frequency Schrödinger operators. Preprint

[AK1] Avila, A., Krikorian, R.: Reducibility or nonuniform hyperbolicity for quasiperiodic Schrödinger cocycles. Ann. of Math. 164, 911-940 (2006) Zbl 1138.47033 MR 2259248

[AK2] Avila, A., Krikorian, R.: Some local and semi-local results for quasiperiodic Schrödinger cocycles. In preparation

[AS] Avron, J., Simon, B.: Almost periodic Schrödinger operators. II. The integrated density of states. Duke Math. J. 50, 369-391 (1983) Zbl 0544.35030 MR 0700145

[BLT] Bellissard, J., Lima, R., Testard, D.: Almost periodic Schrödinger operators. In: Mathematics + Physics. Vol. 1, World Sci., Singapore, 1-64 (1985) Zbl 0675.34022 MR 0849342 
[Be] Berezanskiŭ, Y.: Expansions in Eigenfunctions of Selfadjoint Operators. Transl. Math. Monogr. 17, Amer. Math. Soc., Providence, RI (1968)

[B1] Bourgain, J.: Hölder regularity of integrated density of states for the almost Mathieu operator in a perturbative regime. Lett. Math. Phys. 51, 83-118 (2000) Zbl 0960.34071 MR 1774640

[B2] Bourgain, J.: On the spectrum of lattice Schrödinger operators with deterministic potential. II. J. Anal. Math. 88, 221-254 (2002) Zbl 1058.47002 MR 1984594

[B3] Bourgain, J.: Green's Function Estimates for Lattice Schrödinger Operators and Applications. Ann. of Math. Stud. 158, Princeton Univ. Press, Princeton, NJ (2005) Zbl 1137.35001 MR 2100420

[BJ1] Bourgain, J., Jitomirskaya, S.: Absolutely continuous spectrum for 1D quasiperiodic operators. Invent. Math. 148, 453-463 (2002) Zbl 1036.47019 MR 1908056

[BJ2] Bourgain, J., Jitomirskaya, S.: Continuity of the Lyapunov exponent for quasiperiodic operators with analytic potential. J. Statist. Phys. 108, 1203-1218 (2002) Zbl 1039.81019 MR 1933451

[CL] Carmona, R., Lacroix, J.: Spectral Theory of Random Schrödinger Operators. Birkhäuser, Boston, MA (1990) Zbl 0717.60074 MR 1102675

[CEY] Choi, M. D., Elliott, G. A., Yui, N.: Gauss polynomials and the rotation algebra. Invent. Math. 99, 225-246 (1990) Zbl 0665.46051 MR 1031901

[DJ] De Concini, C., Johnson, R. A.: The algebraic-geometric AKNS potentials. Ergodic Theory Dynam. Systems 7, 1-24 (1987) Zbl 0636.35077 MR 0470318

[DeS] Deift, P., Simon, B.: Almost periodic Schrödinger operators. III. The absolutely continuous spectrum in one dimension. Comm. Math. Phys. 90, 389-411 (1983) Zbl 0562.35026 MR 0719297

[DiS] Dinaburg, E., Sinai, Ya.: The one-dimensional Schrödinger equation with a quasi-periodic potential. Funct. Anal. Appl. 9, 279-289 (1975) Zbl 0333.34014

[E] Eliasson, L. H.: Floquet solutions for the 1-dimensional quasi-periodic Schrödinger equation. Comm. Math. Phys. 146, 447-482 (1992) Zbl $0753.34055 \mid$ MR 1167299

[GS] Goldstein, M., Schlag, W.: Fine properties of the integrated density of states and a quantitative separation property of the Dirichlet eigenvalues. Geom. Funct. Anal. 18, 755-869 (2009) Zbl 1171.82011

[GJLS] Gordon, A. Y., Jitomirskaya, S., Last, Y., Simon, B.: Duality and singular continuous spectrum in the almost Mathieu equation. Acta Math. 178, 169-183 (1997) Zbl 0897.34074 MR 1459260

[H] Herman, M.-R.: Une méthode pour minorer les exposants de Lyapounov et quelques exemples montrant le caractère local d'un théorème d'Arnol'd et de Moser sur le tore de dimension 2. Comment. Math. Helv. 58, 453-502 (1983) Zbl 0554.58034 MR 0727713

[J] Jitomirskaya, S. Ya.: Metal-insulator transition for the almost Mathieu operator. Ann. of Math. (2) 150, 1159-1175 (1999) Zbl 0946.47018 MR 1740982

[JL] Jitomirskaya, S. Ya., Last, Y.: Anderson localization for the almost Mathieu equation. III. Semi-uniform localization, continuity of gaps, and measure of the spectrum. Comm. Math. Phys. 195, 1-14 (1998) Zbl 0922.34074 MR 1637389

[JS] Jitomirskaya, S., Simon, B.: Operators with singular continuous spectrum. III. Almost periodic Schrödinger operators. Comm. Math. Phys. 165, 201-205 (1994) Zbl 0830.34074 MR 1298948

[JM] Johnson, R., Moser, J.: The rotation number for almost periodic potentials. Comm. Math. Phys. 84, 403-438 (1982) Zbl 0497.35026 MR 0667409

[LS] Last, Y., Simon, B.: Eigenfunctions, transfer matrices, and absolutely continuous spectrum of one-dimensional Schrödinger operators. Invent. Math. 135, 329-367 (1999) Zbl 0931.34066 MR 1666767 
[MP] Moser, J., Pöschel, J.: An extension of a result by Dinaburg and Sinai on quasi-periodic potentials. Comment. Math. Helv. 59, 39-85 (1984) Zbl 0533.34023 MR 0743943

[P1] Puig, J.: Cantor spectrum for the almost Mathieu operator. Comm. Math. Phys. 244, 297309 (2004) Zbl 1075.39021 MR 2031032

[P2] Puig, J.: A nonperturbative Eliasson's reducibility theorem. Nonlinearity 19, 355-376 (2006) Zbl 1104.47037 MR 2199393

[S1] Simon, B.: Schrödinger semigroups, Bull. Amer. Math. Soc. 7, 447-526 (1982) Zbl 0524.35002 MR 0670130

[S2] Simon, B.: Schrödinger operators in the twenty-first century. In: Mathematical Physics 2000, Imp. College Press, London, 283-288 (2000) Zbl 1074.81521 MR 1773049 